

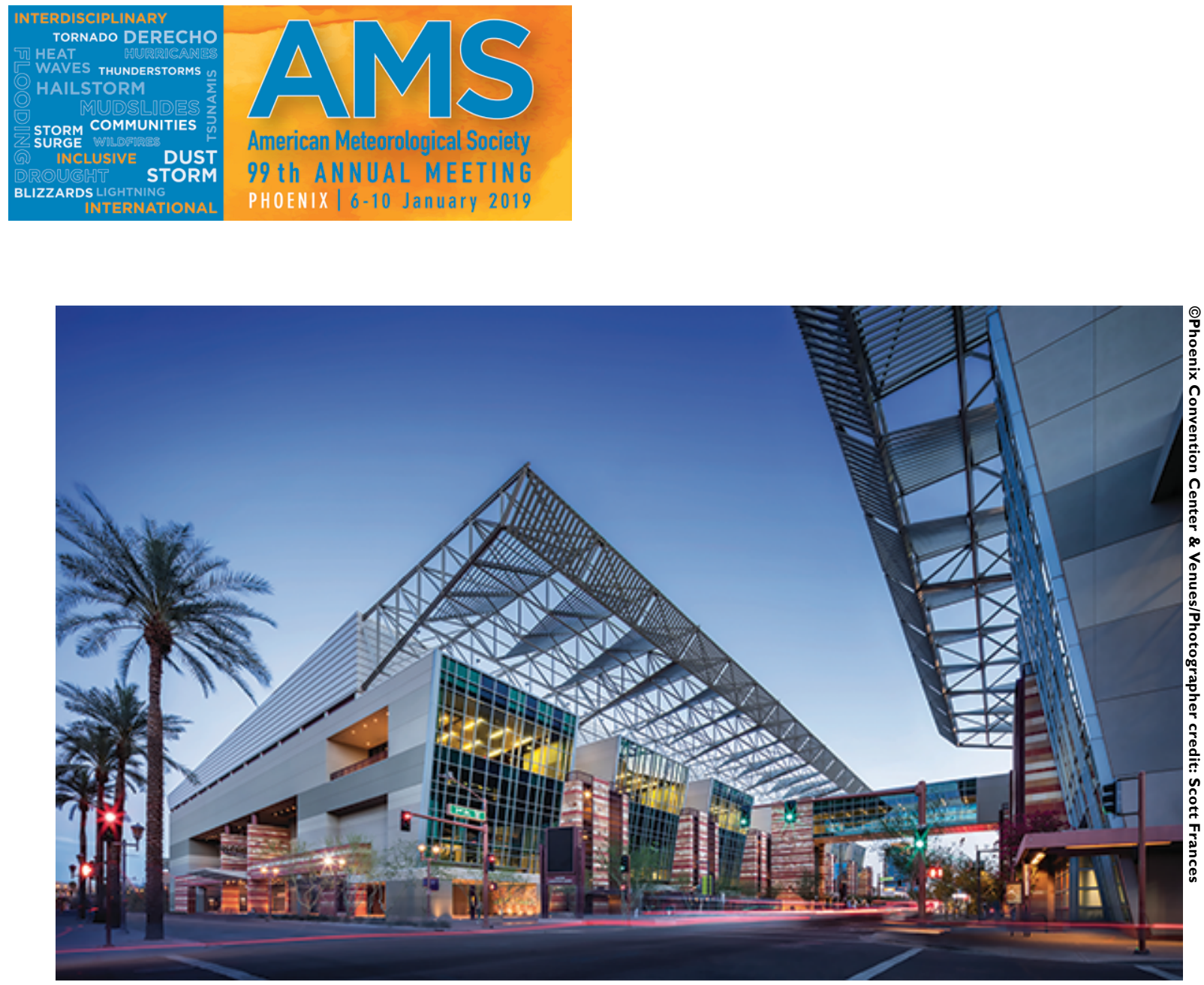

\section{AN INTRODUCTION FROM AMS}

\section{Phoenix Convention Center, Phoenix, Arizona \\ 6-10 January 2019}

MS President Roger Wakimoto and 99th Annual Meeting overall chairpersons, Wen-Chau Lee and
Xubin Zeng, with input from the 99th Annual Meeting program committee and program chairpersons
for each conference, have put together an engaging and informative technical program that will serve as the backbone for this year's meeting. The technical program's content revolves around the 2019 AMS Annual Meeting theme: Understanding and Building Resilience to Extreme Events by Being Interdisciplinary, International, and Inclusive (III). In addition to the technical program, many educational and social events have also been planned to facilitate networking among attendees. The AMS Annual Meeting is also host to the largest exhibit program anywhere in the atmospheric, oceanic, and related sciences. Exhibitors from all over the United States and abroad will be in attendance, with over 100 organizations showcasing a wide range of products, publications, and services.

Please read through the following general information, which serves as a preview for all of the exciting opportunities planned for 99th Annual Meeting attendees, to plan your week in Phoenix. If you have any questions related to the Annual Meeting, please visit the AMS website, www.annual.ametsoc.org/2019, for more information or reach out to the AMS Meetings Department at meetings@ametsoc.org. 


\section{CONFERENCES AND SYMPOSIA}

\section{Listing of Participating Conferences/Symposia}

Conferences and symposia represent the core of the AMS Annual Meeting. They provide the foundation for the organization of our technical sessions. To view the program online, please visit https://ams.confex.com/ams/20I9Annual /meetingapp.cgi/Home/0.

- Presidential Forum

- Richard Anthes Symposium

- Inez Fung Symposium

- 35th Conference on Environmental Information Processing Technologies

- 33rd Conference on Hydrology

- 32nd Conference on Climate Variability and Change

- 28th Symposium on Education

- 24th Conference on Applied Climatology

- 23rd Conference on Integrated Observing and Assimilation Systems for the Atmosphere, Oceans, and Land Surface

- 22nd Conference on Atmospheric Science Librarians International

- 21st Conference on Atmospheric Chemistry

- 20th Conference on Middle Atmosphere

- 19th Conference on Aviation, Range, and Aerospace Meteorology

- 18th Annual AMS Student Conference-Weathering Together: Building a Climate of Diverse Community Perspectives

- 18th Conference on Artificial and Computational Intelligence and Its Applications to the Environmental Sciences

- 17th Symposium on the Coastal Environment

- 17th History Symposium

- 16th Conference on Space Weather

- Major Weather Events and Impacts of 2018

- 15th Annual Symposium on New Generation Operational Environmental Satellite Systems

- 14th Symposium on Societal Applications: Policy, Research, and Practice

- 11th Symposium on Aerosol-Cloud-Climate Interactions

- 10th Conference on Environment and Health

- 10th Conference on Weather, Climate, and the New Energy Economy
- Ninth Conference on the Transition of Research to Operations

- Ninth Symposium on Advances in Modeling and Analysis Using Python

- Ninth Symposium on Lidar Atmospheric Applications

- Ninth Conference on the Meteorological Application of Lightning Data

- Seventh Early Career Professionals Conference

- Seventh Symposium on Building a WeatherReady Nation: Enhancing Our Nation's Readiness, Responsiveness, and Resilience to High Impact Weather Events

- Seventh Symposium on the Weather, Water, and Climate Enterprise

- Seventh Symposium on the Madden-Julian Oscillation and Subseasonal Monsoon Variability

- Seventh AMS Symposium on the Joint Center for Satellite Data Assimilation

- Fifth Symposium on High Performance Computing for Weather, Water, and Climate

- Fourth Symposium on Special Sessions on U.S.-International Partnerships

- Second Conference on Earth Observing SmallSats

- Tropical Cyclones and Extreme Monsoon Precipitation: Prediction, Impacts, and Communication

- Special Symposium on Mesoscale Meteorological Extremes: Understanding, Prediction, and Projection

- Phased Array Radar Symposium

- Special Symposium on Meteorological Observations and Instrumentation

- Special Symposium on Catalyzing Innovation in Weather Science Internationally

- Special Symposium on Global and Mesoscale Models: Updates and Center Overviews

\section{COSPONSORS OF THE 99TH ANNUAL MEETING}

Air \& Waste Management Association

American Academy of Environmental Engineers \& Scientists (AAEES)

American Geophysical Union (AGU)

American Geosciences Institute (AGI)

Australian Meteorological and Oceanographic Society (AMOS)

Canadian Meteorological and Oceanographic Society (CMOS)
Chinese Meteorological Society (CMS)

European Meteorological Society (EMS)

Indian Meteorological Society (IMS)

International Association of Broadcast Meteorology

National Weather Association (NWA) 


\section{9th Presidential Forum and Annual Meeting Welcome: \\ Building Community Resilience to Extreme Political Weather-Advice for Unpredictable Times}

Sunday, 6 January 2019, 4:00 P.M.

AMS is excited to announce the keynote speakers for the upcoming 99th Annual Meeting:

- Dan Kahan: leading science communications and risk perception scholar and Elizabeth K. Dollard Professor of Law and Professor of Psychology at Yale Law School; and

- David Goldston: seasoned U.S. science policy expert and Massachusetts Institute of Technology (MIT) Washington Office Director.

Kahan is one of the nation's leading thinkers and scholars studying the impact of social and cultural values on perceptions of risk and scientific understanding. In studies funded by the National Science Foundation, his research has investigated public disagreement over climate change, public reactions to emerging technologies, and conflicting public impressions of scientific consensus. Prior to coming to Yale University in 1999, Professor Kahan was on the faculty of the University of Chicago Law School. He also served as a law clerk to Justice Thurgood Marshall, of the U.S. Supreme Court, from 1990 to 1991 and to Judge Harry Edwards of the U.S. Court of Appeals for the D.C. Circuit from 1989 to 1990. Kahan is a senior fellow at the National Center for Science and Civic Engagement and an elected member of the American Academy of Arts and Sciences.

Goldston is a seasoned Washington, D.C.-based science and environmental policy expert and advocate. He was chief of staff of the House Science Committee from 2001 to 2006, capping a 20-year career on Capitol Hill. After retiring from government service, Goldston was a visiting lecturer at Princeton University's Woodrow Wilson School of Public and International Affairs and at Harvard University's Center for the Environment, and he is currently an adjunct professor at Georgetown University. From 2007 through November 2009, he wrote a monthly column for Nature on science policy titled "Party of One." Goldston also was the project director for the Bipartisan Policy Center report "Improving the Use of Science in Regulatory Policy," released in August 2009. He authored a chapter in The Science of Science Policy: A Handbook, published in 2011. More recently, Goldston served as director of government affairs at the environmental nonprofit Natural Resources Defense Council. He is a member of the Advisory Committee of the National Academy of Sciences' Division of Environment and Life Sciences and has served on numerous panels of the academy and other science policy organizations.

Please join Roger Wakimoto, AMS President, after the Presidential Forum, for his review of major events from the past year. Each of the AMS Commissioners will give a brief report highlighting the key elements of the many programs and initiatives that are a success due to the efforts of hundreds of volunteers in the Society. All Annual Meeting attendees are invited to the introduction of the newly elected AMS Fellows and the presentation of several featured awards. Jenni Evans will then present her goals and vision for her upcoming year as AMS President.

For more information, visit https://annual.ametsoc.org/index.cfm/2019 /programs/conferences-and-symposia/presidential-forum/.

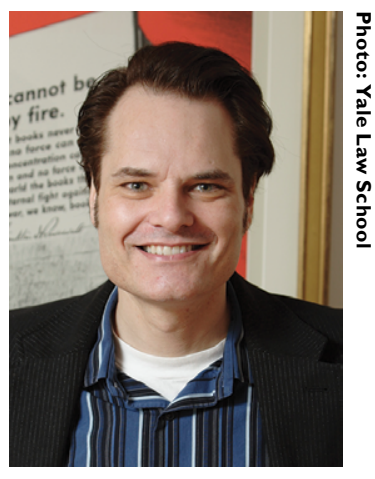

Dan Kahan

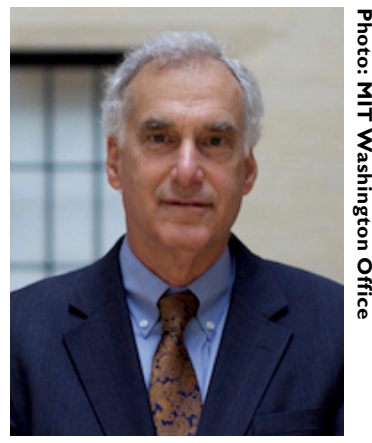

David Goldston

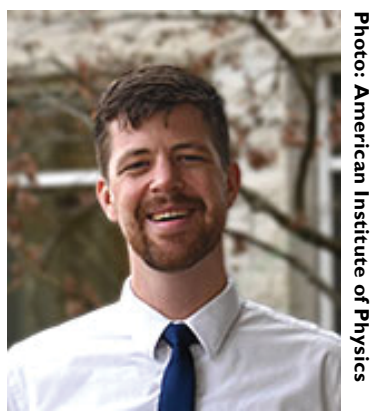

Mike Henry

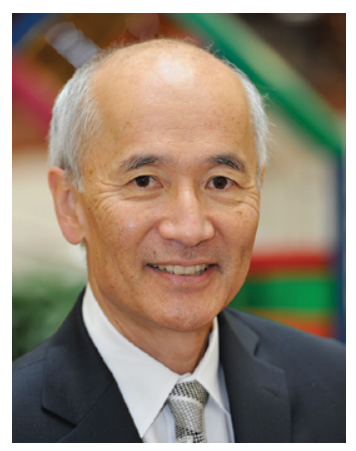

Roger Wakimoto 


\section{Presidential Town Hall Meeting with Marcia McNutt: Charting a Course for Evidence-Based Policy in the Face of "Extreme Events"}

Marcia McNutt, President, National Academy of Sciences

Monday, 7 January 2019, 12:15-I:45 P.M.

The National Academy of Sciences (NAS) was chartered by Congress during the Civil War to provide the nation with evidence-based advice for sound public policy. The Academy has been responding recently to a number of "extreme events" in order to fulfill its mission. The first is that the time scale for decision-makers has rapidly contracted while the problems have become very much more complex. The Academy must become more nimble to provide advice with faster turnaround while breaking down interdisciplinary barriers. The second problem is that the current administration has been historically slow to nominate its political appointees, particularly those in science roles. The good news is that the director of the White House Office of Science and Technology Policy (OSTP) has been nominated and is a meteorologist, of clear interest to the AMS. Finally, the \#MeToo movement has caused a watershed moment for confronting sexual harassment. Fortunately, the National Academies began a study of sexual harassment in academic science, engineering, and medicine about two years ago, and the report was released this summer. The NAS is now acting on the recommendations by convening scientific leadership to change the culture in higher education and professional societies while also initiating changes within the Academy.

Marcia McNutt (B.A. in physics, Colorado College; Ph.D. in Earth sciences, Scripps Institution of Oceanography) is a geophysicist and the 22nd president of the National Academy of Sciences.

From 2013 to 2016, she was editor-in-chief of the journal Science. McNutt was director of the U.S. Geological Survey (USGS) from 2009 to 2013, during which time USGS responded to a number of major disasters, including the Deepwater Horizon oil spill. For her work in helping to contain that spill, McNutt was awarded the U.S. Coast Guard's Meritorious Service Medal.

She is a fellow of the American Geophysical Union (AGU), the Geological Society of America, the American Association for the Advancement of Science, and the International Association of Geodesy. McNutt is a member of the American Philosophical Society and the American Academy of Arts and Sciences, and a Foreign Member of the U.K. Royal Society, and the Russian Academy of Sciences.

In 1998, McNutt was awarded the AGU's Macelwane Medal for research accomplishments by a young scientist, and she received the Maurice Ewing Medal in 2007 for her contributions to deep-sea exploration.

\section{Presidential Town Hall Meeting with Bob Riddaway: Do Meteorological Societies Do Things That Others Cannot?}

\section{Bob Riddaway, President, European Meteorological Society}

Wednesday, 9 January 2019, 12:15-1:15 P.M.

Meteorological societies come in many shapes and sizes. So, for example, some are mainly professional bodies or learned societies that cater for the needs of professionals engaged in meteorology, whereas others embrace the weather enthusiasts who have no professional involvement. Also meteorological societies are engaged in a wide variety of activities such as holding meetings, providing educational activities and resources, publishing journals and/or newsletters, and accrediting meteorologists. But that raises the interesting question about what it is that meteorological societies do that cannot be done by others - what is their "unique selling point". Experience of the Member Societies of the European Meteorological Society will be used to try to address that question.

Bob Riddaway retired from the Met Office in the UK in 2005, though he the continued working part-time at ECMWF for another ten years. After gaining a BSc in Physics and $\mathrm{PhD}$ in Meteorology from Edinburgh University he joined the Met Office to do research. He soon found that training and operational meteorology and were more to his liking, so his career included being Head of Training, Joint Head of Forecasting and Head of Development Resourcing \& Technology. Whilst at the Met Office he became

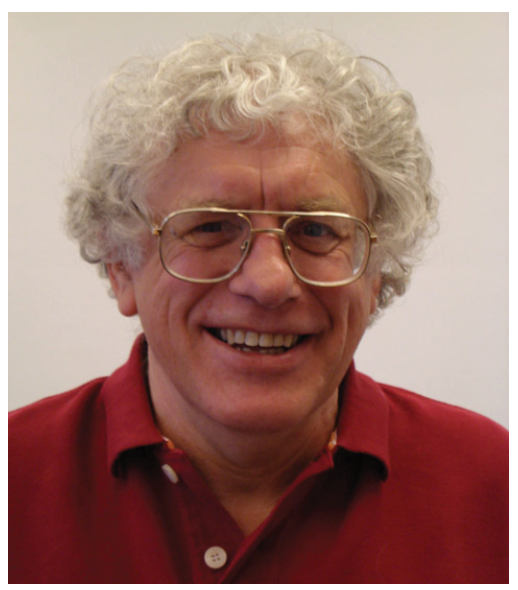
involved in the education and training activities of WMO, and this still continues.

For nearly fifty years Bob Riddaway has been involved in the activities of the Royal Meteorological Society. These included running various educational activities, helping to establish professional meteorological qualifications and being the founding editor of Meteorological Applications. For eight years he was the General Secretary and is currently a member of the Accreditation Board. He became a member of the EMS Council in 2003 and was Vice President between 2008 and 2015. In 2017 he was elected as EMS President. 


\begin{tabular}{|c|c|c|}
\hline Session Title & Host Conference & Day/time \\
\hline $\begin{array}{l}\text { Mind the Gap: Preparing Students for Careers } \\
\text { in the Private Sector }\end{array}$ & 28th Symposium on Education & $\begin{array}{l}\text { Monday, } 7 \text { January } 2019 \text {, } \\
\text { 8:30-10:00 A.M. }\end{array}$ \\
\hline $\begin{array}{l}\text { The Other Uncertainty: Social, Political, and } \\
\text { Cultural Forms of Uncertainty in Weather } \\
\text { Contexts-Part I }\end{array}$ & $\begin{array}{l}\text { 14th Symposium on Societal Applications: } \\
\text { Policy, Research, and Practice }\end{array}$ & $\begin{array}{l}\text { Monday, } 7 \text { January 2019, } \\
\text { 8:30-10:00 A.M. }\end{array}$ \\
\hline $\begin{array}{l}\text { The Other Uncertainty: Social, Political, and } \\
\text { Cultural Forms of Uncertainty in Weather } \\
\text { Contexts-Part II }\end{array}$ & $\begin{array}{l}\text { 14th Symposium on Societal Applications: } \\
\text { Policy, Research, and Practice }\end{array}$ & $\begin{array}{l}\text { Monday, } 7 \text { January } 2019 \text {, } \\
\text { 10:30-12:00 P.M. }\end{array}$ \\
\hline $\begin{array}{l}\text { Role of the Stratosphere in Climate Variability, } \\
\text { Change, and Prediction- Part I }\end{array}$ & $\begin{array}{l}\text { 32nd Conference on Climate Variability and } \\
\text { Change }\end{array}$ & $\begin{array}{l}\text { Monday, } 7 \text { January } 2019 \text {, } \\
\text { 2:00-4:00 P.M. }\end{array}$ \\
\hline $\begin{array}{l}\text { The Fourth National Climate Assessment Vol. II: } \\
\text { Impacts, Risks, and Adaptation in the United States }\end{array}$ & $\begin{array}{l}\text { 14th Symposium on Societal Applications: } \\
\text { Policy, Research, and Practice }\end{array}$ & $\begin{array}{l}\text { Monday, } 7 \text { January } 2019 \text {, } \\
\text { 2:00-4:00 P.M. }\end{array}$ \\
\hline $\begin{array}{l}\text { The Other Uncertainty: Social, Political, and } \\
\text { Cultural Forms of Uncertainty in Weather } \\
\text { Contexts-Part III }\end{array}$ & $\begin{array}{l}\text { I4th Symposium on Societal Applications: } \\
\text { Policy, Research, and Practice }\end{array}$ & $\begin{array}{l}\text { Monday, } 7 \text { January } 2019 \text {, } \\
\text { 2:00-4:00 P.M. }\end{array}$ \\
\hline $\begin{array}{l}\text { Stratospheric Ozone, Chemistry, and Climate- } \\
\text { Part I }\end{array}$ & 20th Conference on Middle Atmosphere & $\begin{array}{l}\text { Tuesday, } 8 \text { January } 2019 \text {, } \\
\text { 8:30-10:00 A.M. }\end{array}$ \\
\hline $\begin{array}{l}\text { National Weather Service Research, Reports, and } \\
\text { Insights to Inform Social Sciences }\end{array}$ & $\begin{array}{l}\text { 14th Symposium on Societal Applications: } \\
\text { Policy, Research, and Practice }\end{array}$ & $\begin{array}{l}\text { Tuesday, } 8 \text { January } 2019 \text {, } \\
\text { 8:30-10:00 A.M. }\end{array}$ \\
\hline $\begin{array}{l}\text { Stratospheric Ozone, Chemistry, and Climate- } \\
\text { Part II }\end{array}$ & 20th Conference on Middle Atmosphere & $\begin{array}{l}\text { Tuesday, } 8 \text { January } 2019 \text {, } \\
\text { 10:30-12:00 P.M. }\end{array}$ \\
\hline $\begin{array}{l}\text { Meteorologist and Emergency Management } \\
\text { Concerns: Public Health, Critical Incident Stress, } \\
\text { and PTSD among Experts in Weather Disasters }\end{array}$ & $\begin{array}{l}\text { 14th Symposium on Societal Applications: } \\
\text { Policy, Research, and Practice }\end{array}$ & $\begin{array}{l}\text { Tuesday, } 8 \text { January } 2019 \text {, } \\
\text { 10:30-12:00 P.M. }\end{array}$ \\
\hline $\begin{array}{l}\text { Too Hot to Handle: Multidisciplinary Perspectives } \\
\text { on Extreme Heat as Disaster-Part I }\end{array}$ & 24th Conference on Applied Climatology & $\begin{array}{l}\text { Tuesday, } 8 \text { January } 2019 \text {, } \\
\text { I:30-2:00 P.M. }\end{array}$ \\
\hline Core Science Keynote on Air Quality Extremes & 2 Ist Conference on Atmospheric Chemistry & $\begin{array}{l}\text { Tuesday, } 8 \text { January } 2019 \text {, } \\
\text { I:30-2:00 P.M. }\end{array}$ \\
\hline $\begin{array}{l}\text { Big Data, Big Computing, Bigger Science: Earth } \\
\text { Data for Al }\end{array}$ & $\begin{array}{l}\text { 18th Conference on Artificial and Computa- } \\
\text { tional Intelligence and Its Applications to the } \\
\text { Environmental Sciences }\end{array}$ & $\begin{array}{l}\text { Tuesday, } 8 \text { January } 2019 \text {, } \\
\text { I:30-2:00 P.M. }\end{array}$ \\
\hline $\begin{array}{l}\text { GOES-16/17 Geostationary Lightning Mapper (GLM) } \\
\text { Sensor Performance, Data Products, and User } \\
\text { Applications-Part I }\end{array}$ & $\begin{array}{l}\text { I5th Annual Symposium on New Generation } \\
\text { Operational } \\
\text { Environmental Satellite Systems }\end{array}$ & $\begin{array}{l}\text { Tuesday, } 8 \text { January } 2019 \text {, } \\
\text { I:30-2:00 P.M. }\end{array}$ \\
\hline $\begin{array}{l}\text { Too Hot to Handle: Multidisciplinary Perspectives } \\
\text { on Extreme Heat as Disaster-Part II }\end{array}$ & 24th Conference on Applied Climatology & $\begin{array}{l}\text { Tuesday, } 8 \text { January } 2019 \text {, } \\
\text { 3:00-4:00 P.M. }\end{array}$ \\
\hline $\begin{array}{l}\text { Role of the Stratosphere in Climate Variability, } \\
\text { Change, and Prediction-Part II }\end{array}$ & 20th Conference on Middle Atmosphere & $\begin{array}{l}\text { Tuesday, } 8 \text { January } 2019 \text {, } \\
\text { 3:00-4:00 P.M. }\end{array}$ \\
\hline $\begin{array}{l}\text { GOES-16/17 Geostationary Lightning Mapper (GLM) } \\
\text { Sensor Performance, Data Products, and User } \\
\text { Applications-Part II }\end{array}$ & $\begin{array}{l}\text { I5th Annual Symposium on New Generation } \\
\text { Operational } \\
\text { Environmental Satellite Systems }\end{array}$ & $\begin{array}{l}\text { Tuesday, } 8 \text { January } 2019 \text {, } \\
\text { 3:00-4:00 P.M. }\end{array}$ \\
\hline $\begin{array}{l}\text { Hurricanes and Health: When Will We Stop } \\
\text { "Learning Lessons" and Start Building Smarter? }\end{array}$ & $\begin{array}{l}\text { Seventh Symposium on Building a Weather- } \\
\text { Ready Nation }\end{array}$ & $\begin{array}{l}\text { Tuesday, } 8 \text { January } 2019 \text {, } \\
\text { 3:00-4:00 P.M. }\end{array}$ \\
\hline $\begin{array}{l}\text { Between the Rock of Applied Needs and the Hard } \\
\text { Place of Diminishing Resources: Rethinking Success } \\
\text { in Provider-Partner Relationships }\end{array}$ & $\begin{array}{l}\text { 14th Symposium on Societal Applications: } \\
\text { Policy, Research, and Practice }\end{array}$ & $\begin{array}{l}\text { Wednesday, } 9 \text { January } 2019 \text {, } \\
\text { 8:30-10:00 A.M. }\end{array}$ \\
\hline $\begin{array}{l}\text { How Can } \mathrm{Al} / \mathrm{Stat} \text { Models Be Interpreted Physically } \\
\text { (joint with the AMS Committee on Probability and } \\
\text { Statistics) }\end{array}$ & $\begin{array}{l}\text { 18th Conference on Artificial and Computa- } \\
\text { tional Intelligence and Its Applications to the } \\
\text { Environmental Sciences }\end{array}$ & $\begin{array}{l}\text { Wednesday, } 9 \text { January } 2019 \text {, } \\
\text { 10:30-12:00 P.M. }\end{array}$ \\
\hline Vulnerability and Extreme Events & $\begin{array}{l}\text { 14th Symposium on Societal Applications: } \\
\text { Policy, Research, and Practice }\end{array}$ & $\begin{array}{l}\text { Wednesday, } 9 \text { January } 2019 \text {, } \\
\text { 10:30-12:00 P.M. }\end{array}$ \\
\hline
\end{tabular}




\begin{tabular}{|c|c|c|}
\hline \multicolumn{3}{|c|}{ THEMED JOINT SESSION SCHEDULE (CONTINUED) } \\
\hline Session Title & Host Conference & Day/time \\
\hline $\begin{array}{l}\text { Extreme Wildfire and Smoke Plumes-Causes, } \\
\text { Impacts, Predictability (themed joint session) }\end{array}$ & $\begin{array}{l}\text { Ninth Symposium on Lidar Atmospheric } \\
\text { Applications }\end{array}$ & $\begin{array}{l}\text { Wednesday, } 9 \text { January } 2019, \\
\text { 10:30-12:00 P.M. }\end{array}$ \\
\hline $\begin{array}{l}\text { Data Stewardship: Finding, Accessing, and Using } \\
\text { Data Online-Part I }\end{array}$ & $\begin{array}{l}\text { 22nd Atmospheric Science Librarians } \\
\text { International Conference }\end{array}$ & $\begin{array}{l}\text { Thursday, } 10 \text { January } 2019 \text {, } \\
\text { 8:30-9:30 A.M. }\end{array}$ \\
\hline $\begin{array}{l}\text { GOES-16/17 Geostationary Lightning Mapper (GLM) } \\
\text { Sensor Performance, Data Products, and User } \\
\text { Applications-Part III }\end{array}$ & $\begin{array}{l}\text { Ninth Conference on the Meteorological } \\
\text { Application of Lightning Data }\end{array}$ & $\begin{array}{l}\text { Thursday, } 10 \text { January } 2019 \text {, } \\
\text { 10:30-12:00 P.M. }\end{array}$ \\
\hline $\begin{array}{l}\text { GOES-16/17 Geostationary Lightning Mapper (GLM) } \\
\text { Sensor Performance, Data Products, and User } \\
\text { Applications-Part IV }\end{array}$ & $\begin{array}{l}\text { Ninth Conference on the Meteorological } \\
\text { Application of Lightning Data }\end{array}$ & $\begin{array}{l}\text { Thursday, } 10 \text { January } 2019 \text {, } \\
\text { I:30-3:00 P.M. }\end{array}$ \\
\hline $\begin{array}{l}\text { Data Stewardship: Finding, Accessing, and Using } \\
\text { Data Online-Part II }\end{array}$ & $\begin{array}{l}\text { 22nd Atmospheric Science Librarians } \\
\text { International Conference }\end{array}$ & $\begin{array}{l}\text { Thursday, } 10 \text { January } 2019 \text {, } \\
\text { I:30-3:00 P.M. }\end{array}$ \\
\hline
\end{tabular}

\section{Special Session: Exceptional Undergraduate Student Presentations}

Tuesday, 8 January 2019, 12:15-I:15 P.M.

Starting this year, select undergraduate presentations from the 18th Annual AMS Student Conference will be featured during the 99th Annual Meeting in a special oral session entitled "Exceptional Undergraduate Student Presentations." This session will feature four highly motivated, talented, and engaging undergraduate students with diverse research backgrounds.

\section{LECTURES}

\section{Walter Orr Roberts Lecture}

Tuesday, 8 January 2019, 1:30-2:30 P.M.

The Walter Orr Roberts Lecture will be given in a session sponsored by the 32nd Conference on Climate Variability and Change. The lecture will be given by John E. Walsh, International Arctic Research Center, University of Alaska, Fairbanks. The title of the lecture is "Arctic sea ice loss: A bellwether challenge to interdisciplinary science."

\section{Robert E. Horton Lecture}

Wednesday, 9 January 2019, 1:30-2:30 P.M.

The Robert E. Horton Lecture will be given in a session sponsored by the 33rd Conference on Hydrology. The lecture will be given by Justin Sheffield, University of Southampton, Southampton, UK. The title of the lecture is "Integrated Approaches for Drought Monitoring and Early Warning in Data Scarce Regions."

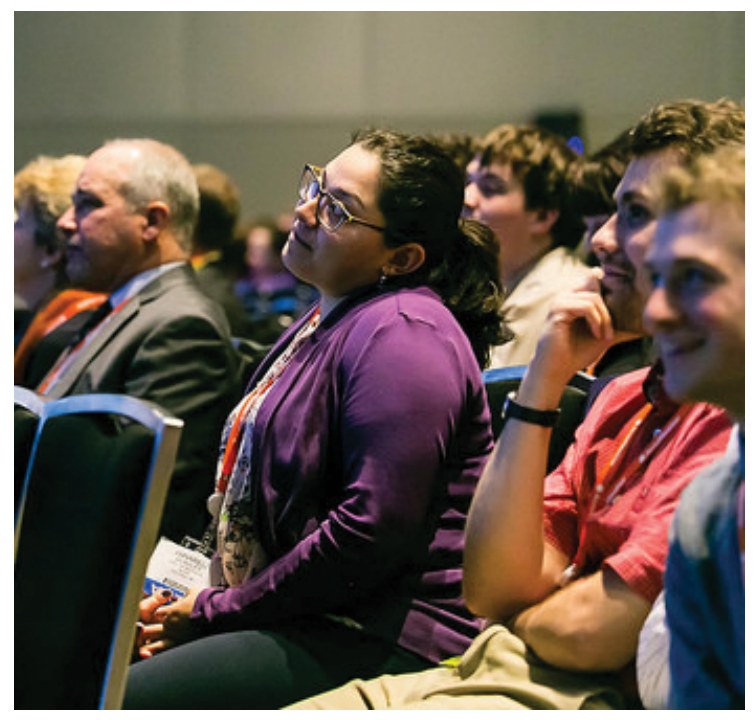




\section{TOWN HALL MEETINGS/SIDE PANEL DISCUSSIONS}

\section{Town Hall/Side Panel Title}

Town hall meeting: DOE's Energy Exascale Earth System Model (E3SM): Atmospheric Model Capabilities and Development Plans

Town hall meeting: NASA's Earth Science Flight Program-Investments in and Planning for the Next-Generation Earth Observatories, with Eric lanson (NASA HQ)

Town hall meeting: Upcoming NCEI Data Users Conference: An Agriculture Example

Town hall meeting: Developing NOAA's FY20192023 R\&D Plan: Public Input Listening Session

Side panel: Building Resiliency to Extreme Weather Events through International Partnerships on the Application of Advanced Satellite Technology

Town hall meeting: Connecting the Forecasting Needs to Solutions of the Analysis and Nowcast (0-18 Hour Forecast) through the Requirements of the National Weather Service

Town hall meeting: EarthCube: A CommunityDriven Cyberinfrastructure for the Geosciences-A Progress Report

Town hall meeting: NCAR/UCAR Strategic Plan

Town hall meeting: NOAA Leadership

Town hall meeting: NASA Earth Science Division (ESD)

Side panel: AMS Publications-Moving into the

Next Century

Town hall meeting: NOAA Big Data Project Updates

Town hall meeting: NOAA Model Development Forum

Town hall meeting: Statistics versus Machine Learning for Complex Problems: White, B lack or Gray Boxes?

Town hall meeting: A Discussion on the Successes and Challenges of Communicating the Science and Data to Users

Town hall meeting: U.S. Air Force Weather Capabilities Roadmap

\section{Day/Time}

Monday, 7 January 2019,

12:15-I:I5 P.M.

For additional information contact Dorothy M

Koch at Dorothy.Koch@science.doe.gov

Monday, 7 January 2019,

I2:15-I:I5 P.M.

A limited number of boxed lunches will be provided by Orbital ATK \& the Northrop Grumman Corporation. For additional information contact Robert Bauer at robert.bauer@nasa. gov

Monday, 7 January 2019, 12:15-I:I5 P.M.

For additional information contact Annette Hollingshead at Annette.hollingshead@noaa.gov

Tuesday, 8 January 2019, For additional information contact Gary C. Mat7:00-8:15 P.M. lock at gary.c.matlock@noaa.gov

Tuesday, 8 January 2019, I2:I5-I:I5 P.M. For additional information contact Gary McWilliams at gary.mcwilliams@noaa.gov

Tuesday, 8 January 2019, I2:15-I:I5 P.M.

For additional information contact Young-Joon Kim at young-joon.kim@noaa.gov

Tuesday, 8 January 2019, 12:15-I:I5 P.M.

Tuesday, 8 January 2019, 12:15-I:I5 P.M.

Tuesday, 8 January 2019 , I2:15-I:I5 P.M.

Tuesday, 8 January 2019, 6:00-7:00 P.M.

Wednesday, 9 January 2019, 12:15-I:I5 P.M.
For additional information contact Mohan K. Ramamurthy at mohan@ucar.edu

For additional information contact James Hurrell at jhurrell@ucar.edu

For additional information contact Timothy Gallaudet at invites.undersecretary@noaa.gov

For additional information contact Jack $A$. Kaye at Jack.A.Kaye@nasa.gov

a limited number of fresh baked cookies will be provided. For additional information contact Michael Friedman at mfriedman@ametsoc.org

Wednesday, 9 January 2019, For additional information contact Nazila Merati 12:15-I:15 P.M.

Wednesday, 9 January 2019, 12:15-I:I5 P.M. at nazila.merati@gmail.com

For additional information contact Hendrik Tolman at Hedrik.Tolman@noaa.gov

Wednesday, 9 January 2019, For additional information contact Elizabeth A. 12:I5-I:I5 P.M. Satterfield at elizabeth.satterfield@nrlmry.navy.mil

Wednesday, 9 January 2019, For additional information contact Kathryn I2:15-I:I5 P.M. Shontz at Kathryn.Shontz@noaa.gov

Wednesday, 9 January 2019, For additional information contact John P. Dreher 12:I5-I:I5 P.M. at john.dreher.I@us.af.mil 


\begin{tabular}{|c|c|c|c|c|}
\hline Keynote Title & $\begin{array}{c}\text { Final } \\
\text { Paper } \\
\text { Number }\end{array}$ & Program Title & Keynote Speaker & Day/Time \\
\hline $\begin{array}{l}\text { Connecting Machine Learning with } \\
\text { Atmospheric Science }\end{array}$ & I.I & $\begin{array}{l}\text { Ninth Symposium on Advances } \\
\text { in Modeling and Analysis Using } \\
\text { Python }\end{array}$ & Rachel Prudden & $\begin{array}{l}\text { Monday, } 7 \text { January } 2019 \text {, } \\
\text { 8:45-9:15 A.M. }\end{array}$ \\
\hline $\begin{array}{l}\text { At Home, Work, and Play: How } \\
\text { Can We Better Understand the } \\
\text { Dynamics between Physiology, } \\
\text { Behavior, and Exposure? }\end{array}$ & Jl.I & $\begin{array}{l}\text { I0th Conference on } \\
\text { Environment and Health }\end{array}$ & Rebekah Lucas & $\begin{array}{l}\text { Monday, } 7 \text { January 2019, } \\
\text { I0:30-10:45 A.M. }\end{array}$ \\
\hline $\begin{array}{l}\text { Using Physiological Evidence } \\
\text { to Inform Extreme Heat Public } \\
\text { Health Guidance }\end{array}$ & Jl.2 & $\begin{array}{l}\text { I0th Conference on } \\
\text { Environment and Health }\end{array}$ & Ollie Jay & $\begin{array}{l}\text { Monday, } 7 \text { January 2019, } \\
\text { 10:45-II:00 A.M. }\end{array}$ \\
\hline $\begin{array}{l}\text { Communicating Uncertainty } \\
\text { in Weather, Climate, and } \\
\text { Hydrological Predictions: Recent } \\
\text { Progress and a Path Forward }\end{array}$ & TJ3.7 & $\begin{array}{l}\text { 14th Symposium on Societal } \\
\text { Applications: Policy, Research, } \\
\text { and Practice }\end{array}$ & Rebecca E. Morss & $\begin{array}{l}\text { Monday, } 7 \text { January } 2019 \text {, } \\
\text { 3:30-4:00 P.M. }\end{array}$ \\
\hline $\begin{array}{l}\text { The } 2018 \text { UNEP/WMO Scientific } \\
\text { Assessment of Ozone Depletion }\end{array}$ & TJ4.I & $\begin{array}{l}\text { 20th Conference on } \\
\text { Middle Atmosphere }\end{array}$ & Paul A. Newman & $\begin{array}{l}\text { Tuesday, } 8 \text { January } 2019 \text {, } \\
\text { 8:30-9:00 A.M. }\end{array}$ \\
\hline $\begin{array}{l}\text { Phased Array Radar Applications } \\
\text { in Cloud and Precipitation } \\
\text { Research }\end{array}$ & I.I & $\begin{array}{l}\text { Phased Array Radar } \\
\text { Symposium }\end{array}$ & Pavlos Kollias & $\begin{array}{l}\text { Tuesday, } 8 \text { January } 2019 \text {, } \\
\text { 8:30-9:00 A.M. }\end{array}$ \\
\hline $\begin{array}{l}\text { A Woman in the Middle: } \\
\text { Communicating the El Niño- } \\
\text { Southern Oscillation }\end{array}$ & 4.1 & $\begin{array}{l}\text { 24th Conference on Applied } \\
\text { Climatology }\end{array}$ & Michelle L'Heureux & $\begin{array}{l}\text { Tuesday, } 8 \text { January } 2019 \text {, } \\
\text { I0:30-II:00 A.M. }\end{array}$ \\
\hline $\begin{array}{l}\text { Findings from Phased Array Radar } \\
\text { Innovative Sensing Experiments }\end{array}$ & 2.1 & $\begin{array}{l}\text { Phased Array Radar } \\
\text { Symposium }\end{array}$ & Pamela L. Heinselman & $\begin{array}{l}\text { Tuesday, } 8 \text { January } 2019 \text {, } \\
\text { 10:30-II:00 A.M. }\end{array}$ \\
\hline Al for Earth & TJI0.I & $\begin{array}{l}\text { 18th Conference on Artificial } \\
\text { and Computational Intelligence } \\
\text { and Its Applications to the } \\
\text { Environmental Sciences }\end{array}$ & Lucas Joppa & $\begin{array}{l}\text { Tuesday, } 8 \text { January } 2019 \text {, } \\
\text { I:30-2:00 P.M. }\end{array}$ \\
\hline $\begin{array}{l}\text { Can Air Pollution Fuel Extreme } \\
\text { Weather Events and Contribute } \\
\text { to Long-Term Climate Changes? }\end{array}$ & TJ9.1 & $\begin{array}{l}\text { 2Ist Conference on } \\
\text { Atmospheric Chemistry }\end{array}$ & Zhanqing Li & $\begin{array}{l}\text { Tuesday, } 8 \text { January } 2019 \text {, } \\
\text { I:30-2:00 P.M. }\end{array}$ \\
\hline $\begin{array}{l}\text { Health Effects of Air Pollutants: A } \\
\text { Future Perspective }\end{array}$ & TJ9.2 & $\begin{array}{l}\text { 2Ist Conference on } \\
\text { Atmospheric Chemistry }\end{array}$ & Tong Zhu & $\begin{array}{l}\text { Tuesday, } 8 \text { January } 2019 \text {, } \\
\text { 2:00-2:30 P.M. }\end{array}$ \\
\hline $\begin{array}{l}\text { Making the Black Box More Trans- } \\
\text { parent: Understanding the Physical } \\
\text { Implications of Machine Learning }\end{array}$ & TJI7.I & $\begin{array}{l}\text { 18th Conference on Artificial } \\
\text { and Computational Intelligence } \\
\text { and Its Applications to the } \\
\text { Environmental Sciences }\end{array}$ & Amy McGovern & $\begin{array}{l}\text { Wednesday, } 9 \text { January } 2019 \text {, } \\
\text { 10:30-II:00 A.M. }\end{array}$ \\
\hline $\begin{array}{l}\text { Climate Impact of Anthropogenic } \\
\text { Aerosols on Large-Scale Cirrus } \\
\text { Clouds }\end{array}$ & 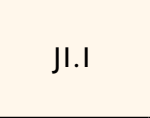 & $\begin{array}{l}\text { Ilth Symposium on Aerosol- } \\
\text { Cloud-Climate Interactions }\end{array}$ & Joyce E. Penner & $\begin{array}{l}\text { Wednesday, } 9 \text { January } 2019 \text {, } \\
\text { 3:00-3:30 P.M. }\end{array}$ \\
\hline $\begin{array}{l}\text { Constraints on Desert Dust } \\
\text { Impacts on Climate Obtained by } \\
\text { Leveraging Understanding of the } \\
\text { Physics of Dust emission }\end{array}$ & Jl.2 & $\begin{array}{l}\text { Ilth Symposium on Aerosol- } \\
\text { Cloud-Climate Interactions }\end{array}$ & Jasper F. Kok & $\begin{array}{l}\text { Wednesday, } 9 \text { January 2019, } \\
\text { 3:30-4:00 P.M. }\end{array}$ \\
\hline
\end{tabular}




\section{AWARD WINNERS}

In an effort to highlight the AMS 2019 Awardees, this honored group was invited to participate in the Annual Meeting. Congratulations to the award winners.

\begin{tabular}{|c|c|c|c|c|}
\hline Award Winner & Program Title & $\begin{array}{c}\text { Final } \\
\text { Paper } \\
\text { Number }\end{array}$ & Paper Title & $\begin{array}{l}\text { Session Day, } \\
\text { Paper Start-End Time }\end{array}$ \\
\hline Peter C. Banacos & $\begin{array}{l}\text { Ninth Conference on Transition } \\
\text { of Research to Operations }\end{array}$ & 5B. 3 & $\begin{array}{l}\text { R2O: Development of NWS } \\
\text { Snow Squall Warnings }\end{array}$ & $\begin{array}{l}\text { Tuesday, } 8 \text { January } 2019 \text {, } \\
\text { II:00-II:15 A.M. }\end{array}$ \\
\hline Stephen W. Bieda & $\begin{array}{l}\text { Seventh Symposium on Building } \\
\text { a Weather-Ready Nation }\end{array}$ & 6.2 & $\begin{array}{l}\text { The Evolving Role of the NWS } \\
\text { Science and Operations Officer: } \\
\text { Preparing Offices on New } \\
\text { Methods of Hazardous Weather } \\
\text { Communication }\end{array}$ & $\begin{array}{l}\text { Wednesday, } 9 \text { January 2019, } \\
\text { I:45-2:00 P.M. }\end{array}$ \\
\hline Suzana J. Camargo & $\begin{array}{l}\text { Tropical Cyclones and Extreme } \\
\text { Monsoon Precipitation: } \\
\text { Prediction, Impacts, and } \\
\text { Communication }\end{array}$ & J5.I & $\begin{array}{l}\text { Dependence of Intraseasonal } \\
\text { Tropical Cyclone Prediction on } \\
\text { the MJO in the S2S Dataset }\end{array}$ & $\begin{array}{l}\text { Tuesday, } 8 \text { January } 2019 \text {, } \\
\text { 10:30-10:45 A.M. }\end{array}$ \\
\hline Jerome P. Charba & $\begin{array}{l}\text { Ninth Conference on the } \\
\text { Meteorological Application of } \\
\text { Lightning Data }\end{array}$ & 8.1 & $\begin{array}{l}\text { Climatological Properties of } \\
\text { Reported Cloud-to-Ground } \\
\text { Lightning for Alaska from Several } \\
\text { Lightning Locating Systems }\end{array}$ & $\begin{array}{l}\text { Thursday, } 10 \text { January } 2019 \text {, } \\
\text { 8:30-8:45 A.M. }\end{array}$ \\
\hline Fei Chen & 33rd Conference on Hydrology & $8 A .5$ & $\begin{array}{l}\text { Challenges in Representing } \\
\text { Hydroclimatic Effects of } \\
\text { Agriculture Management in Earth } \\
\text { System Models }\end{array}$ & $\begin{array}{l}\text { Wednesday, } 9 \text { January 2019, } \\
\text { 9:30-9:45 A.M. }\end{array}$ \\
\hline Haonan Chen & $\begin{array}{l}\text { 35th Conference on } \\
\text { Environmental Information } \\
\text { Processing Technologies }\end{array}$ & & $\begin{array}{l}\text { An Improved Attenuation } \\
\text { Correction Methodology for } \\
\text { Polarimetric Weather Radar }\end{array}$ & $\begin{array}{l}\text { Wednesday, } 9 \text { January 2019, } \\
\text { 4:00-6:00 P.M. }\end{array}$ \\
\hline Haonan Chen & 33rd Conference on Hydrology & & $\begin{array}{l}\text { Representing Radar Rainfall } \\
\text { Uncertainties in Complex } \\
\text { Terrain Using A Bayesian } \\
\text { Modelling Approach }\end{array}$ & $\begin{array}{l}\text { Wednesday, } 9 \text { January } 2019 \text {, } \\
\text { 4:00-6:00 P.M. }\end{array}$ \\
\hline Haonan Chen & $\begin{array}{l}\text { 35th Conference on } \\
\text { Environmental Information } \\
\text { Processing Technologies }\end{array}$ & & $\begin{array}{l}\text { Improving Quantitative } \\
\text { Precipitation Estimation in } \\
\text { Complex Terrain over the } \\
\text { San Francisco Bay Area Using } \\
\text { Gap-Filling Radar Network }\end{array}$ & $\begin{array}{l}\text { Wednesday, } 9 \text { January 2019, } \\
\text { 4:00-6:00 P.M. }\end{array}$ \\
\hline Christopher A. Davis & $\begin{array}{l}\text { Special Symposium on Meso- } \\
\text { scale Meteorological Extremes: } \\
\text { Understanding, Prediction, and } \\
\text { Projection }\end{array}$ & I.I & $\begin{array}{l}\text { Mesoscale Meteorology: } \\
\text { Connecting Weather and } \\
\text { Climate Research }\end{array}$ & $\begin{array}{l}\text { Monday, } 7 \text { January } 2019 \text {, } \\
\text { 8:45-9:15 A.M. }\end{array}$ \\
\hline Christopher A. Davis & $\begin{array}{l}\text { Special Symposium on } \\
\text { Catalyzing Innovation in } \\
\text { Weather Science Internationally }\end{array}$ & I.I & $\begin{array}{l}\text { The Advent of Convection- } \\
\text { Permitting Forecast Models: } \\
\text { Now What? }\end{array}$ & $\begin{array}{l}\text { Tuesday, } 8 \text { January } 2019 \text {, } \\
\text { 8:30-9:00 A.M. }\end{array}$ \\
\hline Gina M. Eosco & $\begin{array}{l}\text { Seventh Symposium on the } \\
\text { Weather, Water, and Climate } \\
\text { Enterprise }\end{array}$ & & $\begin{array}{l}\text { What's That Worth? How to Use } \\
\text { Social, Behavioral, and Economic } \\
\text { Science Methods to Understand } \\
\text { the Value of Earth Observations }\end{array}$ & $\begin{array}{l}\text { Monday, } 7 \text { January 2019, } \\
\text { 4:00-6:00 P.M. }\end{array}$ \\
\hline Gina M. Eosco & $\begin{array}{l}\text { I4th Symposium on Societal } \\
\text { Applications: Policy, Research, } \\
\text { and Practice }\end{array}$ & 9.4 & $\begin{array}{l}\text { Demystifying Social and } \\
\text { Behavioral Science Research } \\
\text { to Application Transitions }\end{array}$ & $\begin{array}{l}\text { Wednesday, } 9 \text { January 2019, } \\
\text { 2:15-2:30 P.M. }\end{array}$ \\
\hline Gina M. Eosco & $\begin{array}{l}\text { 14th Symposium on Societal } \\
\text { Applications: Policy, Research, } \\
\text { and Practice }\end{array}$ & PD4.I & $\begin{array}{l}\text { Coffee Talk: A Social, Behavioral, } \\
\text { and Economic Science Research } \\
\text { and Operations Roundtable } \\
\text { Discussion }\end{array}$ & $\begin{array}{l}\text { Tuesday, } 8 \text { January } 2019 \text {, } \\
\text { 3:00-3:30 P.M. }\end{array}$ \\
\hline
\end{tabular}




\begin{tabular}{|c|c|c|c|c|}
\hline Award Winner & Program Title & $\begin{array}{c}\text { Final } \\
\text { Paper } \\
\text { Number }\end{array}$ & Paper Title & $\begin{array}{l}\text { Session Day, } \\
\text { Paper Start-End Time }\end{array}$ \\
\hline Gina M. Eosco & $\begin{array}{l}\text { Special Symposium on } \\
\text { Catalyzing Innovation in Weath- } \\
\text { er Science Internationally }\end{array}$ & 1.3 & $\begin{array}{l}\text { Using Social and Behavioral } \\
\text { Science Research to Navigate } \\
\text { and Innovate Weather Science } \\
\text { Advancements }\end{array}$ & $\begin{array}{l}\text { Tuesday, } 8 \text { January } 2019 \text {, } \\
\text { 9:30-10:00 A.M. }\end{array}$ \\
\hline M. N. Evans & $\begin{array}{l}\text { 32nd Conference on Climate } \\
\text { Variability and Change }\end{array}$ & 4B. 4 & $\begin{array}{l}\text { Reconstruction and Attribution } \\
\text { of Multidecadal Temperature } \\
\text { Variability over the Common Era }\end{array}$ & $\begin{array}{l}\text { Tuesday, } 8 \text { January } 2019 \text {, } \\
\text { 9:15-9:30 A.M. }\end{array}$ \\
\hline Inez Fung & 33rd Conference on Hydrology & J3.I & $\begin{array}{l}\text { On Transpiration Seasonality } \\
\text { (invited presentation) }\end{array}$ & $\begin{array}{l}\text { Tuesday, } 8 \text { January } 2019 \text {, } \\
\text { I:30-I:45 P.M. }\end{array}$ \\
\hline Inez Fung & Inez Fung Symposium & 4.1 & $\begin{array}{l}\text { Some Thoughts on Projecting } \\
\text { Biosphere-Climate Interactions }\end{array}$ & $\begin{array}{l}\text { Tuesday, } 8 \text { January } 2019 \text {, } \\
\text { 3:45-4:00 P.M. }\end{array}$ \\
\hline Kenneth Graham & $\begin{array}{l}\text { Global and Regional-Scale } \\
\text { Models: Updates and Center } \\
\text { Overviews }\end{array}$ & 3.3 & $\begin{array}{l}\text { A Brief History of and Future } \\
\text { Vision for the Use of Model } \\
\text { Guidance for National Hurricane } \\
\text { Center Forecast Products }\end{array}$ & $\begin{array}{l}\text { Wednesday, } 9 \text { January 2019, } \\
\text { 2:00-2:15 P.M. }\end{array}$ \\
\hline Samson Hagos & $\begin{array}{l}\text { Seventh Symposium on the } \\
\text { Madden-Julian Oscillation } \\
\text { and Subseasonal Monsoon } \\
\text { Variability }\end{array}$ & 2.1 & $\begin{array}{l}\text { A Stochastic Transition Matrix } \\
\text { Approach to Modeling of the } \\
\text { Population Dynamics of Clouds }\end{array}$ & $\begin{array}{l}\text { Monday, } 7 \text { January } 2019 \text {, } \\
\text { 10:30-10:45 A.M. }\end{array}$ \\
\hline Heather A. Holmes & 33rd Conference on Hydrology & $\mathrm{J} 2.3$ & $\begin{array}{l}\text { Land Use Impacts on Surface } \\
\text { Fluxes during Persistent Cold } \\
\text { Air Pool Events }\end{array}$ & $\begin{array}{l}\text { Tuesday, } 8 \text { January } 2019 \text {, } \\
\text { II:00-II:15 A.M. }\end{array}$ \\
\hline Fei-Fei Jin & $\begin{array}{l}\text { 32nd Conference on Climate } \\
\text { Variability and Change }\end{array}$ & $12 \mathrm{C} .2$ & Dynamics of ENSO Diversity & $\begin{array}{l}\text { Thursday, } 10 \text { January } 2019 \text {, } \\
8: 45-9: 00 \text { A.M }\end{array}$ \\
\hline Daryl T. Kleist & $\begin{array}{l}\text { 23rd Conference on Integrated } \\
\text { Observing and Assimilation } \\
\text { Systems for the Atmosphere, } \\
\text { Oceans, and Land Surface }\end{array}$ & 2.3 & $\begin{array}{l}\text { Scale-Dependent Weighting } \\
\text { and Localization for Global } \\
\text { Numerical Weather Prediction }\end{array}$ & $\begin{array}{l}\text { Monday, } 7 \text { January } 2019 \text {, } \\
\text { II:00-II:15 A.M. }\end{array}$ \\
\hline Daryl T. Kleist & $\begin{array}{l}\text { Global and Regional-Scale } \\
\text { Models: Updates and Center } \\
\text { Overviews }\end{array}$ & 5.2 & $\begin{array}{l}\text { Data Assimilation Planning } \\
\text { and Development for Global } \\
\text { and High-Resolution Regional } \\
\text { Numerical Weather Prediction } \\
\text { at NCEP/EMC }\end{array}$ & $\begin{array}{l}\text { Thursday, } 10 \text { January } 2019 \text {, } \\
\text { 8:45-9:00 A.M. }\end{array}$ \\
\hline Jasper F. Kok & $\begin{array}{l}\text { Ilth Symposium on Aerosol- } \\
\text { Cloud-Climate Interactions }\end{array}$ & Jl.2 & $\begin{array}{l}\text { Constraints on Desert Dust } \\
\text { Impacts on Climate Obtained by } \\
\text { Leveraging Understanding of the } \\
\text { Physics } \\
\text { of Dust Emission }\end{array}$ & $\begin{array}{l}\text { Wednesday, } 9 \text { January } 2019 \text {, } \\
\text { 3:30-4:00 P.M. }\end{array}$ \\
\hline Renee A. McPherson & $\begin{array}{l}\text { 24th Conference on Applied } \\
\text { Climatology }\end{array}$ & 4.3 & $\begin{array}{l}\text { Trusted Communication through } \\
\text { a Diverse Workforce: Are You } \\
\text { Serious About It or Just Talking? }\end{array}$ & $\begin{array}{l}\text { Tuesday, } 8 \text { January } 2019 \text {, } \\
\text { II:I5-II:30 A.M. }\end{array}$ \\
\hline Renee A. McPherson & $\begin{array}{l}\text { 32nd Conference on Climate } \\
\text { Variability and Change }\end{array}$ & IOB.I & $\begin{array}{l}\text { Users Perspectives on S2S } \\
\text { Forecast Products for Extreme } \\
\text { Precipitation Events }\end{array}$ & $\begin{array}{l}\text { Wednesday, } 9 \text { January } 2019 \text {, } \\
\text { I:30-I:45 P.M. }\end{array}$ \\
\hline Patrick Minnis & $\begin{array}{l}\text { I5th Annual Symposium on } \\
\text { New Generation Opera- } \\
\text { tional Environmental Satellite } \\
\text { Systems }\end{array}$ & IIB.I & $\begin{array}{l}\text { Cloud Property Retrievals for } \\
\text { CERES from Suomi NPP and JPSS- } \\
\text { I VIIRS to Continue a Long-Term } \\
\text { Cloud and Radiation Climate Data } \\
\text { Record }\end{array}$ & $\begin{array}{l}\text { Thursday, } 10 \text { January } 2019 \text {, } \\
\text { 3:30-4:00 P.M. }\end{array}$ \\
\hline Ada Monzón & $\begin{array}{l}\text { Seventh Symposium on Building } \\
\text { a Weather-Ready Nation }\end{array}$ & 1.2 & $\begin{array}{l}\text { Resiliency through Innovation in } \\
\text { Education: Using STEM to Build } \\
\text { Resilient Communities }\end{array}$ & $\begin{array}{l}\text { Tuesday, } 8 \text { January } 2019 \text {, } \\
\text { 8:45-9:00 A.M. }\end{array}$ \\
\hline Hugh Morrison & 33rd Conference on Hydrology & 13.2 & $\begin{array}{l}\text { Unifying Raindrop Size } \\
\text { Distribution Normalization } \\
\text { Methods Using a New } \\
\text { Generalized Approach }\end{array}$ & $\begin{array}{l}\text { Thursday, } 10 \text { January } 2019 \text {, } \\
\text { I:45-2:00 P.M. }\end{array}$ \\
\hline
\end{tabular}




\begin{tabular}{|c|c|c|c|c|}
\hline Award Winner & Program Title & $\begin{array}{c}\text { Final } \\
\text { Paper } \\
\text { Number }\end{array}$ & Paper Title & $\begin{array}{l}\text { Session Day, } \\
\text { Paper Start-End Time }\end{array}$ \\
\hline J. David Neelin & $\begin{array}{l}\text { Seventh Symposium on the } \\
\text { Madden-Julian Oscillation } \\
\text { and Subseasonal Monsoon } \\
\text { Variability }\end{array}$ & 2.2 & $\begin{array}{l}\text { Controls on Deep-Convective } \\
\text { Precipitation and the Dialogue } \\
\text { with the Mesoscale }\end{array}$ & $\begin{array}{l}\text { Monday, } 7 \text { January 2019, } \\
\text { 10:45-11:00 A.M. }\end{array}$ \\
\hline Bart Nijssen & 33rd Conference on Hydrology & 2B.6 & $\begin{array}{l}\text { From Model Performance } \\
\text { to Model Behavior through } \\
\text { Fingerprinting of Hydrologic } \\
\text { Models (invited presentation) }\end{array}$ & $\begin{array}{l}\text { Monday, } 7 \text { January } 2019 \text {, } \\
\text { II:45-12:00 P.M. }\end{array}$ \\
\hline John Quagliariello & $\begin{array}{l}\text { Seventh Symposium on Building } \\
\text { a Weather-Ready Nation }\end{array}$ & 1.3 & $\begin{array}{l}\text { NWS Support of South Carolina } \\
\text { during Hurricanes Matthew and } \\
\text { Irma }\end{array}$ & $\begin{array}{l}\text { Tuesday, } 8 \text { January } 2019 \text {, } \\
\text { 9:00-9:15 A.M. }\end{array}$ \\
\hline Mark D. Schwartz & Inez Fung Symposium & & $\begin{array}{l}\text { Calculating Synoptic to Global- } \\
\text { Scale Spring Phenology }\end{array}$ & $\begin{array}{l}\text { Tuesday, } 8 \text { January } 2019 \text {, } \\
\text { 4:00-6:00 P.M. }\end{array}$ \\
\hline James A. Smith & 33rd Conference on Hydrology & 13.1 & $\begin{array}{l}\text { How Hard Can It Rain? } \\
\text { (invited presentation) }\end{array}$ & $\begin{array}{l}\text { Thursday, } 10 \text { January } 2019 \text {, } \\
\text { I:30-I:45 P.M. }\end{array}$ \\
\hline David J. Stensrud & $\begin{array}{l}\text { Special Symposium } \\
\text { on Catalyzing Innova- } \\
\text { tion in Weather Science } \\
\text { Internationally }\end{array}$ & 2.1 & $\begin{array}{l}\text { Improvements and Challenges in } \\
\text { High-Impact Weather Prediction }\end{array}$ & $\begin{array}{l}\text { Tuesday, } 8 \text { January } 2019 \text {, } \\
\text { 10:30-11:00 A.M. }\end{array}$ \\
\hline Wassila M. Thiaw & $\begin{array}{l}\text { 32nd Conference on Climate } \\
\text { Variability and Change }\end{array}$ & I3A.2 & $\begin{array}{l}\text { On the Equatorial East Africa } \\
\text { Floods during the Spring } 2018 \\
\text { Rainfall Season }\end{array}$ & $\begin{array}{l}\text { Thursday, } 10 \text { January } 2019 \text {, } \\
\text { 10:45-II:00 A.M. }\end{array}$ \\
\hline Wassila M. Thiaw & $\begin{array}{l}\text { 10th Conference on } \\
\text { Environment and Health }\end{array}$ & 2.6 & $\begin{array}{l}\text { Real-Time Cimate Information } \\
\text { for Heat-Health Early Warning } \\
\text { for Africa }\end{array}$ & $\begin{array}{l}\text { Tuesday, } 8 \text { January } 2019 \text {, } \\
11: 45-12: 00 \text { P.M. }\end{array}$ \\
\hline Wassila M. Thiaw & 33rd Conference on Hydrology & j9.1 & $\begin{array}{l}\text { The Use of Seasonal Performance } \\
\text { Probability in Monitoring and } \\
\text { Predicting Drought in Africa } \\
\text { (invited presentation) }\end{array}$ & $\begin{array}{l}\text { Thursday, } 10 \text { January } 2019 \text {, } \\
\text { 3:30-3:45 P.M. }\end{array}$ \\
\hline Wassila M. Thiaw & $\begin{array}{l}\text { Special Symposium on } \\
\text { Catalyzing Innovation } \\
\text { in Weather Science } \\
\text { Internationally }\end{array}$ & 1.2 & $\begin{array}{l}\text { Operational Predictions of } \\
\text { Extreme Events in Africa and } \\
\text { Decision Support Services }\end{array}$ & $\begin{array}{l}\text { Tuesday, } 8 \text { January } 2019 \text {, } \\
\text { 9:00-9:30 A.M. }\end{array}$ \\
\hline Robert Thompson & 17th History Symposium & 1.6 & $\begin{array}{l}\text { Evolution of Weather } \\
\text { Forecasting-Retrospective } \\
\text { Look by a Retired Operational } \\
\text { Meteorologist }\end{array}$ & $\begin{array}{l}\text { Monday, } 7 \text { January 2019, } \\
\text { 9:45-10:00 A.M. }\end{array}$ \\
\hline Stanley B. Trier & $\begin{array}{l}\text { 19th Conference on Aviation, } \\
\text { Range, and Aerospace } \\
\text { Meteorology }\end{array}$ & 5.6 & $\begin{array}{l}\text { High-Resolution Nested WRF } \\
\text { Simulations of Mechanisms } \\
\text { Contributing to a Widespread } \\
\text { Turbulence Outbreak in Early } \\
\text { Spring }\end{array}$ & $\begin{array}{l}\text { Tuesday, } 8 \text { January } 2019 \text {, } \\
\text { II:45-12:00 P.M. }\end{array}$ \\
\hline
\end{tabular}




\section{HIGHLIGHTED PRESENTATIONS}

\section{NEW FELLOWS}

In an effort to highlight the AMS 2019 Awardees, we invited this honored group to participate in the Annual Meeting. All Annual Meeting attendees are invited to attend their presentations. Congratulations to all of the New Fellows.

\begin{tabular}{|c|c|c|c|c|}
\hline New Fellow & Program Title & $\begin{array}{l}\text { Final } \\
\text { Paper } \\
\text { Number }\end{array}$ & Paper Title & $\begin{array}{l}\text { Session Day, } \\
\text { Paper Start-End Time }\end{array}$ \\
\hline Scott A. Braun & $\begin{array}{l}\text { Tropical Cyclones and } \\
\text { Extreme Monsoon Precipita- } \\
\text { tion: Prediction, Impacts, and } \\
\text { Communication }\end{array}$ & 2.1 & $\begin{array}{l}\text { NASA Space-Based Precipita- } \\
\text { tion Measurements of Tropical } \\
\text { Cyclones: Past, Present, and } \\
\text { Future (invited presentation) }\end{array}$ & $\begin{array}{l}\text { Tuesday, } 8 \text { January } 2019 \text {, } \\
\text { I:30-I:45 P.M. }\end{array}$ \\
\hline Leo Donner & $\begin{array}{l}\text { Ilth Symposium on Aerosol- } \\
\text { Cloud-Climate Interactions }\end{array}$ & 10.1 & $\begin{array}{l}\text { Strong Buffering of Aerosol- } \\
\text { Cloud Interactions by Cloud } \\
\text { Dynamics in Climate Models } \\
\text { (invited presentation) }\end{array}$ & $\begin{array}{l}\text { Thursday, } 10 \text { January } 2019 \text {, } \\
\text { I0:30-10:45 A.M. }\end{array}$ \\
\hline David Gochis & 33rd Conference on Hydrology & 5.2 & $\begin{array}{l}\text { Assessment of Operational and } \\
\text { Retrospective NOAA National } \\
\text { Water Model Performance for } \\
\text { Low-Frequency Flooding Events: } \\
\text { National Synthesis and Case } \\
\text { Studies }\end{array}$ & $\begin{array}{l}\text { Tuesday, } 8 \text { January } 2019 \text {, } \\
\text { I0:45-II:00 A.M. }\end{array}$ \\
\hline Mitchell D. Goldberg & $\begin{array}{l}\text { I5th Annual Symposium on } \\
\text { New Generation Operational } \\
\text { Environmental Satellite Systems }\end{array}$ & 1.1 & $\begin{array}{l}\text { The Evolution of the Joint Polar } \\
\text { Satellite System: How Polar } \\
\text { Satellites Transformed Weather } \\
\text { Forecasting }\end{array}$ & $\begin{array}{l}\text { Monday, } 7 \text { January } 2019 \text {, } \\
\text { 8:30-9:00 A.M. }\end{array}$ \\
\hline Sue Ellen Haupt & $\begin{array}{l}\text { I0th Conference on Weather, } \\
\text { Climate, and the New Energy } \\
\text { Economy }\end{array}$ & J3.1 & $\begin{array}{l}\text { Artificial Intelligence and Meteo- } \\
\text { rology Empowering Renewable } \\
\text { Energy }\end{array}$ & $\begin{array}{l}\text { Thursday, } 10 \text { January } 2019 \text {, } \\
\text { I0:30-II:00 A.M. }\end{array}$ \\
\hline Gabriele Hegerl & $\begin{array}{l}\text { 32nd Conference on Climate } \\
\text { Variability and Change }\end{array}$ & 8.5 & $\begin{array}{l}\text { Attributing Causes to "Old" } \\
\text { Extremes }\end{array}$ & $\begin{array}{l}\text { Wednesday, } 9 \text { January 2019, } \\
\text { 9:30-9:45 A.M. }\end{array}$ \\
\hline George J. Huffman & 33rd Conference on Hydrology & IIA.I & $\begin{array}{l}\text { Reaching for } 20 \text { Years with the } \\
\text { IMERG Multisatellite Products }\end{array}$ & $\begin{array}{l}\text { Thursday, } 10 \text { January } 2019 \text {, } \\
\text { 8:30-8:45 A.M. }\end{array}$ \\
\hline George J. Huffman & 33rd Conference on Hydrology & 14.1 & $\begin{array}{l}\text { The IMERG Experience in Building } \\
\text { Precipitation Products that Users } \\
\text { Want (invited presentation) }\end{array}$ & $\begin{array}{l}\text { Thursday, } 10 \text { January } 2019 \text {, } \\
\text { 3:30-3:45 P.M. }\end{array}$ \\
\hline Fei-Fei Jin & $\begin{array}{l}\text { 32nd Conference on Climate } \\
\text { Variability and Change }\end{array}$ & I2C.2 & Dynamics of ENSO Diversity & $\begin{array}{l}\text { Thursday, } 10 \text { January } 2019 \text {, } \\
\text { 8:45-9:00 A.M. }\end{array}$ \\
\hline Ben Kirtman & $\begin{array}{l}\text { Seventh Symposium on the } \\
\text { Weather, Water, and Climate } \\
\text { Enterprise }\end{array}$ & 2.1 & $\begin{array}{l}\text { The NMME and SubX Proj- } \\
\text { ects: Status, Challenges, and } \\
\text { Opportunities (invited presenta- } \\
\text { tion) }\end{array}$ & $\begin{array}{l}\text { Wednesday, } 9 \text { January 2019, } \\
\text { I0:30-10:45 A.M. }\end{array}$ \\
\hline Ben Kirtman & $\begin{array}{l}\text { 32nd Conference on Climate } \\
\text { Variability and Change }\end{array}$ & J5.6 & $\begin{array}{l}\text { The Subseasonal Prediction } \\
\text { Experiment }\end{array}$ & $\begin{array}{l}\text { Wednesday, } 9 \text { January } 2019 \text {, } \\
9: 45-10: 00 \text { A.M. }\end{array}$ \\
\hline Delores J. Knipp & $\begin{array}{l}\text { 16th Conference on Space } \\
\text { Weather }\end{array}$ & 8.1 & $\begin{array}{l}\text { The Last Gasp of Solar Cycle } \\
20 \text { and the Little-Known } \\
\text { Consequences of the August } \\
1972 \text { Ultra-Fast Coronal Mass } \\
\text { Ejecta (invited presentation) }\end{array}$ & $\begin{array}{l}\text { Wednesday, } 9 \text { January 2019, } \\
\text { 8:30-9:15 A.M. }\end{array}$ \\
\hline Praveen Kumar & 33rd Conference on Hydrology & 2B.I & $\begin{array}{l}\text { Using Information Flow } \\
\text { Networks for Characterizing } \\
\text { Process and Model Dynamics } \\
\text { (invited presentation) }\end{array}$ & $\begin{array}{l}\text { Monday, } 7 \text { January } 2019 \text {, } \\
\text { 10:30-10:45 A.M. }\end{array}$ \\
\hline Gary M. Lackmann & $\begin{array}{l}\text { Ninth Conference on Transi- } \\
\text { tion of Research to Operations }\end{array}$ & I4A.I & $\begin{array}{l}\text { A Case for Operational Utility in } \\
\text { Nontraditional NWP Output Fields }\end{array}$ & $\begin{array}{l}\text { Thursday, } 10 \text { January } 2019 \text {, } \\
\text { I:30-I:45 P.M. }\end{array}$ \\
\hline
\end{tabular}




\section{NEW FELLOWS (CONTINUED)}

\begin{tabular}{|c|c|c|c|c|}
\hline New Fellow & Program Title & $\begin{array}{l}\text { Final } \\
\text { Paper } \\
\text { Number }\end{array}$ & Paper Title & $\begin{array}{l}\text { Session Day, } \\
\text { Paper Start-End Time }\end{array}$ \\
\hline Shawn W. Miller & $\begin{array}{l}\text { I5th Annual Symposium on } \\
\text { New Generation Operational } \\
\text { Environmental Satellite Systems }\end{array}$ & 7A.I & $\begin{array}{l}\text { Modernization of the Weather } \\
\text { Value Chain }\end{array}$ & $\begin{array}{l}\text { Wednesday, } 9 \text { January 2019, } \\
\text { 3:00-3:30 P.M. }\end{array}$ \\
\hline Michael C. Morgan & $\begin{array}{l}\text { Tropical Cyclones and } \\
\text { Extreme Monsoon Precipita- } \\
\text { tion: Prediction, Impacts, and } \\
\text { Communication }\end{array}$ & 2.2 & $\begin{array}{l}\text { Adjoint Sensitivity Diagnosis of } \\
\text { Hurricane Irma (2017) Intensity } \\
\text { Change }\end{array}$ & $\begin{array}{l}\text { Tuesday, } 8 \text { January } 2019 \text {, } \\
\text { I:45-2:00 P.M. }\end{array}$ \\
\hline Rebecca E. Morss & $\begin{array}{l}\text { Tropical Cyclones and } \\
\text { Extreme Monsoon Precipita- } \\
\text { tion: Prediction, Impacts, and } \\
\text { Communication }\end{array}$ & 5.1 & $\begin{array}{l}\text { Hurricane Risk Communication and } \\
\text { Decision-Making in the Modern } \\
\text { Information Environment (invited } \\
\text { presentation) }\end{array}$ & $\begin{array}{l}\text { Wednesday, } 9 \text { January 2019, } \\
\text { I0:30-10:45 A.M. }\end{array}$ \\
\hline Rebecca E. Morss & $\begin{array}{l}\text { 14th Symposium on Societal } \\
\text { Applications: Policy, Research, } \\
\text { and Practice }\end{array}$ & TJ3.7 & $\begin{array}{l}\text { Communicating Uncertainty } \\
\text { in Weather, Climate, and } \\
\text { Hydrological Predictions: Recent } \\
\text { Progress and a Path Forward } \\
\text { (Core Science Keynote) }\end{array}$ & $\begin{array}{l}\text { Monday, } 7 \text { January } 2019 \text {, } \\
\text { 3:30-4:00 P.M. }\end{array}$ \\
\hline Mike Nelson & $\begin{array}{l}\text { 32nd Conference on Climate } \\
\text { Variability and Change }\end{array}$ & I2B.I & $\begin{array}{l}\text { Communicating Climate Change- } \\
\text { Be the Expert in the Living Room }\end{array}$ & $\begin{array}{l}\text { Thursday, } 10 \text { January } 2019 \text {, } \\
\text { 8:30-8:45 A.M. }\end{array}$ \\
\hline T. N. Palmer & Inez Fung Symposium & 2.4 & $\begin{array}{l}\text { Climate Communication and } \\
\text { Next-Generation Climate Models }\end{array}$ & $\begin{array}{l}\text { Tuesday, } 8 \text { January } 2019, \\
\text { II:I5-II:30 A.M. }\end{array}$ \\
\hline T. N. Palmer & $\begin{array}{l}\text { Special Symposium on } \\
\text { Catalyzing Innovation in } \\
\text { Weather Science Internationally }\end{array}$ & 3.1 & $\begin{array}{l}\text { Proactive Response to Forecasts } \\
\text { of Natural Disasters: The Role of } \\
\text { Ensemble Prediction }\end{array}$ & $\begin{array}{l}\text { Tuesday, } 8 \text { January } 2019 \text {, } \\
\text { I:30-2:00 P.M. }\end{array}$ \\
\hline Joyce E. Penner & $\begin{array}{l}\text { Ilth Symposium on Aerosol- } \\
\text { Cloud-Climate Interactions }\end{array}$ & JI.I & $\begin{array}{l}\text { Climate Impact of Anthropogenic } \\
\text { Aerosols on Large-Scale Cirrus } \\
\text { Clouds }\end{array}$ & $\begin{array}{l}\text { Wednesday, } 9 \text { January 2019, } \\
\text { 3:00-3:30 P.M. }\end{array}$ \\
\hline Joyce E. Penner & $\begin{array}{l}\text { 2Ist Conference on Atmo- } \\
\text { spheric Chemistry }\end{array}$ & $8 A .2$ & $\begin{array}{l}\text { The Effects of New Particle } \\
\text { Formation by Products Associated } \\
\text { with the Oxidation of } \alpha \text {-pinene }\end{array}$ & $\begin{array}{l}\text { Wednesday, } 9 \text { January } 2019 \text {, } \\
\text { I:45-2:00 P.M. }\end{array}$ \\
\hline Zhaoxia Pu & $\begin{array}{l}\text { 23rd Conference on Integrated } \\
\text { Observing and Assimilation } \\
\text { Systems for the Atmosphere, } \\
\text { Oceans, and Land Surface }\end{array}$ & 12.3 & $\begin{array}{l}\text { Advances in Wind Measurements } \\
\text { and Their Impacts on Severe } \\
\text { Weather Forecasting: Progress } \\
\text { and Prospects }\end{array}$ & $\begin{array}{l}\text { Wednesday, } 9 \text { January 2019, } \\
\text { 2:00-2:15 P.M. }\end{array}$ \\
\hline Zhaoxia Pu & $\begin{array}{l}\text { Ninth Symposium on Lidar } \\
\text { Atmospheric Applications }\end{array}$ & 10.1 & $\begin{array}{l}\text { Assimilation of Wind Data from } \\
\text { the Doppler Aerosol Wind } \\
\text { (DAWN) to Improve the Numeri- } \\
\text { cal Simulations and Understanding } \\
\text { of Convective Systems over the } \\
\text { Gulf of Mexico and Its Vicinity }\end{array}$ & $\begin{array}{l}\text { Thursday, } 10 \text { January } 2019 \text {, } \\
\text { 10:30-10:45 A.M. }\end{array}$ \\
\hline Zhaoxia Pu & $\begin{array}{l}\text { Seventh Symposium on the } \\
\text { Madden-Julian Oscillation and } \\
\text { Subseasonal Monsoon Variability }\end{array}$ & I.I & $\begin{array}{l}\text { Characteristics of Diurnal Convec- } \\
\text { tion and Winds over Maritime } \\
\text { Continent during YMC with WRF } \\
\text { Numerical Simulations and Data } \\
\text { Assimilation at a Cloud Permitting } \\
\text { Scale }\end{array}$ & $\begin{array}{l}\text { Monday, } 7 \text { January } 2019 \text {, } \\
\text { 8:30-8:45 A.M. }\end{array}$ \\
\hline Timothy J. Schmit & $\begin{array}{l}\text { I5th Annual Symposium on } \\
\text { New Generation Operational } \\
\text { Environmental Satellite Systems }\end{array}$ & $4 \mathrm{~A} . \mathrm{I}$ & $\begin{array}{l}\text { Monitoring Tropical Cyclones from } \\
\text { Satellite: improvements from } 1966 \\
\text { to } 2018\end{array}$ & $\begin{array}{l}\text { Wednesday, } 9 \text { January 2019, } \\
\text { 8:30-9:00 A.M. }\end{array}$ \\
\hline
\end{tabular}




\begin{tabular}{|c|c|c|c|c|}
\hline NEW FELL & S (CONTINUED) & $\begin{array}{l}\text { Final } \\
\text { Paper } \\
\text { Number }\end{array}$ & Paper Title & $\begin{array}{l}\text { Session Day, } \\
\text { Paper Start-End Time }\end{array}$ \\
\hline David M. Schultz & 28th Symposium on Education & 1.5 & $\begin{array}{l}\text { Build Your Own Earth: A Climate } \\
\text { and Paleoclimate Tool for Teaching } \\
\text { and Research }\end{array}$ & $\begin{array}{l}\text { Monday, } 7 \text { January 2019, } \\
\text { 10:33-10:36 A.M. }\end{array}$ \\
\hline David M. Schultz & $\begin{array}{l}\text { I0th Conference on } \\
\text { Environment and Health }\end{array}$ & $\mathrm{J} 2.8$ & $\begin{array}{l}\text { The Relationship between Weath- } \\
\text { er and Pain Severity: Results from } \\
\text { Cloudy with a Chance of Pain, A } \\
\text { U.K. National Smartphone Study }\end{array}$ & $\begin{array}{l}\text { Monday, } 7 \text { January 2019, } \\
\text { 3:45-4:00 P.M. }\end{array}$ \\
\hline David M. Schultz & $\begin{array}{l}\text { Special Symposium on } \\
\text { Mesoscale Meteorological } \\
\text { Extremes: Understanding, } \\
\text { Prediction, and Projection }\end{array}$ & J4.I & $\begin{array}{l}\text { Strong Winds in Extratropical } \\
\text { Cyclones: Sting Jets and Cold } \\
\text { Conveyor Belts }\end{array}$ & $\begin{array}{l}\text { Wednesday, } 9 \text { January } 2019 \text {, } \\
\text { I:30-I:45 P.M. }\end{array}$ \\
\hline Rong Zhang & $\begin{array}{l}\text { 32nd Conference on Climate } \\
\text { Variability and Change }\end{array}$ & 4B.I & $\begin{array}{l}\text { Underestimated Atlantic Me- } \\
\text { ridional Overturning Circulation } \\
\text { (AMOC) Variability and Implica- } \\
\text { tions for Atlantic Multidecadal } \\
\text { Variability (AMV) and Predictabil- } \\
\text { ity in CMIP Models }\end{array}$ & $\begin{array}{l}\text { Tuesday, } 8 \text { January 2019, } \\
\text { 8:30-8:45 A.M. }\end{array}$ \\
\hline
\end{tabular}

\section{SHORT COURSES/WORKSHOPS}

Please note that short courses/workshops require registration and fees that are separate from the 99th Annual Meeting registration.

\section{SHORT COURSES}

Friday, 5 January 2019

\begin{tabular}{|c|c|c|}
\hline 8:00 A.M. $-5: 00$ P.M. & Room $221 \mathrm{C}$ & Integrating NWP System Components Using Container Technology \\
\hline 8:30 A.M. $-5: 00$ P.M. & Room 226C & Tutorial on How to Build a 3D-Printed Automatic Weather Station \\
\hline 8:30A.M. $-5: 30$ P.M. & Room 22IAB & A Beginner's Course to Using Python in Climate and Meteorology \\
\hline \multicolumn{3}{|c|}{ Saturday, 6 January 2019} \\
\hline 7:30 A.M. $-2: 30$ P.M. & Room 22IAB & A Beginner's Course to Using Python in Climate and Meteorology \\
\hline 8:00 A.M. $-12: 00$ P.M. & Room 225AB & Weather and Phased Array Radar Polarimetry \\
\hline 8:00 A.M. $-3: 45$ P.M. & Room 224A & $\begin{array}{l}\text { An Introduction to Using the NASA Giovanni System for Multidisciplinary Research } \\
\text { and Applications }\end{array}$ \\
\hline 8:00 A.M. $-3: 45$ P.M. & Room 222C & Applying JPSS Data Products to Better Forecast Challenging Weather Events \\
\hline 8:00 A.M. $-12: 00$ P.M. & Room 226AB & $\begin{array}{l}\text { Introducing the Community Earth System Model (CESM2) Via an Interactive } \\
\text { Hands-on Tutorial Using the Cloud. }\end{array}$ \\
\hline 8:00 A.M.-3:45 P.M. & Room 222AB & GOES-R Series: Forecasting Applications \\
\hline 8:00 A.M.-3:45 P.M. & Room 228AB & Diversity, Equity \& Inclusion for Geoscientists \\
\hline 8:30 A.M. $-3: 45$ P.M. & Room 22IC & AMS Short Course on Python for Synoptic Meteorology Using Metpy \\
\hline 8:30 A.M. $-3: 45$ P.M. & Room 223 & Machine Learning in Python for Environmental Science Problems \\
\hline 8:30 A.M.-12:30 P.M. & Room 230 & $\begin{array}{l}\text { Using NASA High Performance Computers to Perform Analytics on Large Earth } \\
\text { Science Data }\end{array}$ \\
\hline
\end{tabular}




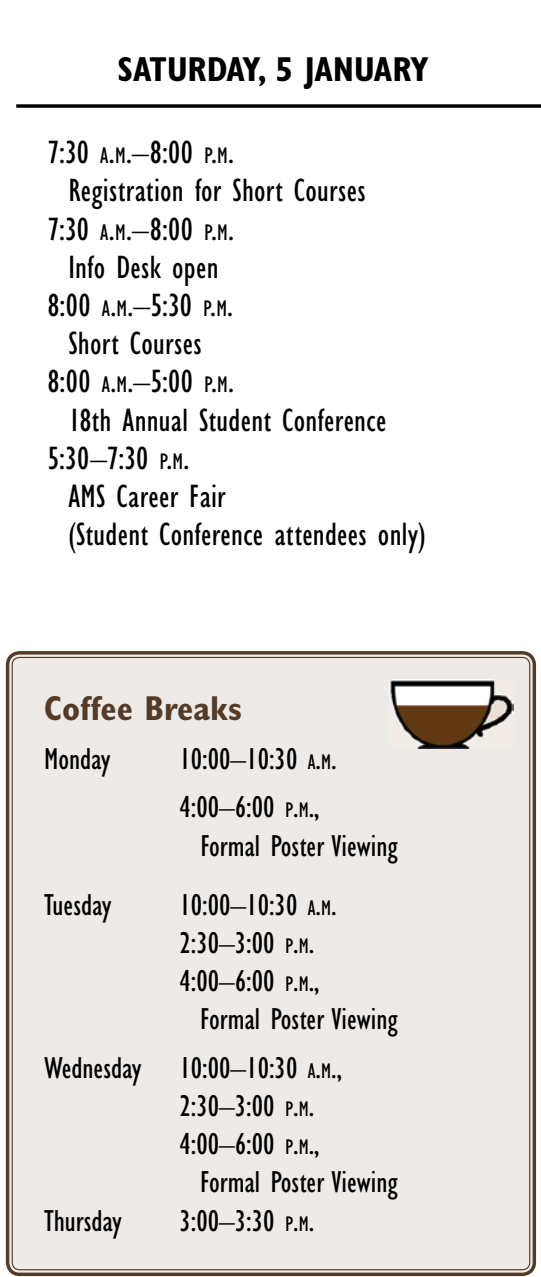

\section{GET THE 99TH ANNUAL MEETING MOBILE APP}

Get the most from your 2019 AMS 99th Annual Meeting experience by getting the AMS mobile app!

Visit the AMS website in early December for instructions on how to download the latest mobile app.
SUNDAY, 6 JANUARY

MONDAY, 7 JANUARY

7:00 A.M. $-12: 00$ P.M.

WxReady Scout Event

7:00 A.M. $-8: 30$ P.M.

Registration open

7:30 A.M.-8:30 P.M.

Info Desk open

7:30 A.M.-3:45 P.M.

Short Courses

8:30 A.M. $-9: 00$ A.M.

Meet the President and Presidential Forum

Speakers (for students and early career professionals)

9:00 A.M. $-3: 45$ P.M.

18th Annual Student Conference

9:00 A.M.-3:45 P.M.

Seventh Conference for Early Career

Professionals

12:00-3:45 P.M.

Speaker Ready Room open

12:00-8:30 P.M.

Poster Hall open

2:30-3:30 P.M.

Newcomer's Welcome and Informational Exchange

4:00-6:30 P.M.

Presidential Forum and Annual Meeting Welcome

6:30-8:30 P.M.

AMS Career Fair

6:30-8:30 P.M.

Student Conference Poster Session

6:30-7:30 P.M.

Welcome Reception Honoring Newly Elected

Fellows and Featured Award Winners

8:00-10:00 P.M.

CoRiolis Reception

8:30-10:00 P.M.

Colour of Weather Networking Reception 9:00-II:00 P.M.

Early Career Professional Reception
7:30 A.M. $-6: 00$ P.M. Registration open

7:30 A.M. $-6: 00$ P.M.

Info Desk open

7:30 A.M. $-6: 00$ P.M.

Quiet Room open

7:30 A.M. $-6: 00$ P.M.

Speaker Ready Room open

9:00 A.M.-7:30 P.M.

Poster Hall open

8:30.-10:00 A.M.

Technical Sessions

9:00.-II:00 A.M.

Guest Coffee

10:00 A.M.-6:00 P.M.

Member Services Desk open

10:00-10:30 A.M.

Morning Coffee Break

10:30 A.M.-12:00 P.M.

Technical Sessions

12:00-1:30 P.M.

Lunch Break

12:15-1:45 P.M.

Presidential Town Hall Meeting with

Marcia McNutt

12:15-I:I5 P.M.

Town Hall Meetings and Side Panels

1:30-2:30 P.M.

How to Become a Better Presenter 2:00-4:00 P.M.

Technical Sessions

4:00-6:00 P.M.

Formal Poster Viewing Reception 6:00-8:00 P.M.

Ribbon Cutting, Exhibits Opening Reception, and Corporate Patron Recognition 8:00-10:00 P.M.

Speed Networking Event for Students and Early Career Professionals 
7:00-8:15 A.M.

Town Hall Meetings and Side Panels

7:30 A.M. $-6: 00$ P.M.

Registration open

7:30 A.M. $-6: 00$ P.M.

Info Desk open

7:30 A.M. $-6: 00$ P.M.

Member Services Desk open

7:30 A.M. $-6: 00$ P.M.

Quiet Room open

7:30 A.M. $-6: 00$ P.M.

Speaker Ready Room open

8:30 A.M. $-4: 00$ P.M.

Inez Fung Symposium

8:30-10:00 A.M.

Technical Sessions

9:00 A.M.-6:00 P.M.

Poster Hall open

9:00 A.M.-6:00 P.M.

Exhibit Hall open

10:00-10:30 A.M.

Morning Coffee Break

10:00-10:30 A.M.

Meet the President

10:30-II:00 A.M.

Technical Sessions

I2:00-I:30 P.M.

Lunch Break

12:00-1:30 P.M.

Women in the Atmospheric Sciences

Luncheon

12:15-I:15 P.M.

Town Hall Meetings and Side Panels

12:15-I:15 P.M.

Exceptional Undergraduate Presentations

12:45-I:05 P.M.

Daily Weather Briefing

1:30-2:30 P.M.

Technical Sessions

2:30-3:00 P.M.

Afternoon Coffee Break

3:00-4:00 P.M.

Technical Sessions

4:00-6:00 P.M.

Formal Poster Viewing Reception

4:00-6:00 P.M.

Book Talks at the Authors' Corner 6:00-7:00 P.M.

Town Hall Meetings and Side Panels

6:00-10:00 P.M.

University Night Receptions

6:30-8:30 P.M.

Inez Fung Dinner
7:30 A.M. $-6: 00$ P.M.

Registration open

7:30 A.M. $-6: 00$ P.M.

Info Desk open

7:30 A.M.-6:00 P.M.

Member Services Desk open

7:30 A.M.-6:00 P.M.

Quiet Room open

7:30 A.M.-6:00 P.M.

Speaker Ready Room open

9:00 A.M.-6:30 P.M.

Poster Hall open

8:30 A.M.-10:00 A.M.

Technical Sessions

8:30 A.M. $-4: 00$ P.M.

Richard Anthes Symposium

9:00 A.M. $-6: 30$ P.M.

Exhibit Hall open

10:00-10:30 A.M.

Morning Coffee Break

10:00-10:30 A.M.

Meet the President

10:30-12:00 P.M.

Technical Sessions

12:00-I:30 P.M.

Lunch Break

12:15-I:15 P.M.

Town Hall Meetings and Side Panels

12:15-I:15 P.M.

Presidential Town Hall Meeting with

Bob Riddaway

12:00-I:30 P.M.

Richard Anthes Luncheon

12:45-I:05 P.M.

Daily Weather Briefing

I:30-2:30 P.M.

Robert E. Horton Lecture

I:30-2:30 P.M.

Technical Sessions

2:30-3:00 P.M.

Afternoon Coffee Break

3:00-4:00 P.M.

Technical Sessions

4:00-6:00 P.M.

Formal Poster Viewing Reception

4:00-6:00 P.M.

Book Talks at the Authors' Corner 6:00-6:30 P.M.

Awards Banquet Reception

7:00-10:00 P.M.

99th AMS Awards Banquet
7:30 A.M. $-3: 00$ P.M.

Registration open

7:30 A.M.-3:00 P.M.

Info Desk open

7:30 A.M.-3:00 P.M.

Member Services Desk open

7:30 A.M.-3:00 P.M.

Quiet Room open

7:30 A.M.-5:00 P.M.

Speaker Ready Room open

8:30-9:30 A.M.

Technical Sessions

9:00 A.M. $-12: 00$ P.M.

Exhibit Hall open

9:30-10:30 A.M.

Exhibit Hall Breakfast

10:00-10:30 A.M.

Meet the President

10:30 A.M.-12:00 P.M.

Technical Sessions

12:00-I:30 P.M.

Lunch Break

12:15-I:15 P.M.

Town Hall Meetings and Side Panels

12:45-I:05 P.M.

Daily Weather Briefing

1:00-4:00 P.M.

Tour of Riparian Preserve at Water Ranch

1:30-3:00 P.M.

Technical Sessions

1:30-2:30 P.M.

Walter Orr Robert Lecture

3:00-3:30 P.M.

Afternoon Coffee Break

3:30-5:00 P.M.

Technical Sessions

5:00 P.M.

Meeting Adjourns 


\title{
OFFICERS OF THE 99TH ANNUAL MEETING
}

\section{OVERALL PLANNING COMMITTEE}

Wen Chau Lee and Xubin Zeng, Cochairpersons

\author{
Julie Demuth \\ Rebecca Hacker
}

Christopher Schultz

Sarah Jones

PROGRAM CHAIRPERSONS

$\begin{array}{lllll}\text { Angel Adames-Corraliza } & \text { Jerry Crescenti } & \text { Michael Jamilkowski } & \text { Stephen Mango } & \text { Owen Shieh } \\ \text { Philip Ardanuy } & \text { Sean Davis } & \text { Jonathan Jiang } & \text { Amy McGovern } & \text { Tim Sliwinski } \\ \text { Bob Atlas } & \text { Ken Dewey } & \text { Michael Johnson } & \text { Gary McWilliams } & \text { Jennifer Sprague } \\ \text { Tom Auligne } & \text { Sheldon Drobot } & \text { Dave Jones } & \text { Eric Miller } & \text { Geoffrey Stano } \\ \text { Lourdes B. Avilés } & \text { Kacey Earst } & \text { Hunter Jones } & \text { Ottmar Moehler } & \text { Vijay Tallapragada } \\ \text { Jane Baldwin } & \text { Eric Fetzer } & \text { Sarah Jones } & \text { Kathryn Mozer } & \text { Barbara Thompson } \\ \text { Robert Banks } & \text { Matthew Flournoy } & \text { Satya Kalluri } & \text { Gretchen Mullendore } & \text { Philippe Tissot } \\ \text { Robert Bauer } & \text { Trent Ford } & \text { Emil Kepco } & \text { Jinny Nathans } & \text { Sara Tucker } \\ \text { Tom Bedard } & \text { Tanja Fransen } & \text { Gaige Kerr } & \text { Jennifer Newman } & \text { Tiffany Vance } \\ \text { Rich Behnke } & \text { Rong Fu } & \text { Young-Joon Kim } & \text { Kenneth Pickering } & \text { Andre Van der Westhuysen } \\ \text { Reginald Blake } & \text { David John Gagne } & \text { Jason Knievel } & \text { James Pinto } & \text { Jennifer Vanos } \\ \text { David Bludis } & \text { Tom Galarneau } & \text { Chandra Kondragunta } & \text { Scott Powell } & \text { Vanessa Vincente } \\ \text { David Bodine } & \text { Larry Gloeckler } & \text { Rob Korty } & \text { Jared Rennie } & \text { Zhuo Wang } \\ \text { Amy Butler } & \text { Mitch Goldberg } & \text { Makenzie Krocak } & \text { Walt Robinson } & \text { Klaus Wolter } \\ \text { James Campbell } & \text { Elise Gowen } & \text { Sujay Kumar } & \text { Joshua Roundy } & \text { Chun-Chieh Wu } \\ \text { Margaret Caulfield } & \text { Jen Henderson } & \text { Bill Kuo } & \text { Paolo Ruti } & \text { Martin Yapur } \\ \text { Rich Clark } & \text { Douglas Hilderbrand } & \text { Scott Landolt } & \text { Naoko Sakaeda } & \text { Jim Yoe } \\ \text { Chris Coleman } & \text { Mike Hobbins } & \text { Heather Lazrus } & \text { Timothy J. Schmit } & \text { John Yorks } \\ \text { Jeff Collett } & \text { Adele lgel } & \text { Renee Leduc Clarke } & \text { Carl Schueler } & \text { Jeffrey Yuhas } \\ \text { Scott Collis } & \text { Scott Jacobs } & \text { Sharan Majumdar } & \text { Justin Sharp } & \text { Nusrat Yussouf } \\ \text { Gerry Creager } & & & & \end{array}$

\section{TOUR OF RIPARIAN PRESERVE AT WATER RANCH} THURSDAY, I0 JANUARY 2019, I:00 P.M.-4:00 P.M.

\section{Sponsored by AMS Committee on Environmental Stewardship}

Thursday, 10 January 2019, 2:00-3:30 P.M. (weather permitting), $\$ 25$ fee

$$
\begin{array}{cl}
\text { I:00 P.M. } & \text { Shuttle bus departs from } \\
& \text { Phoenix Convention Center } \\
\text { 4:00 P.M. } & \text { Return trip arrives at } \\
& \text { Phoenix Convention Center }
\end{array}
$$

ocated in Gilbert, Arizona, just southeast of Phoenix, the

Riparian Preserve is a II0-acre site that combines water resource development, wildlife habitat, and educational and

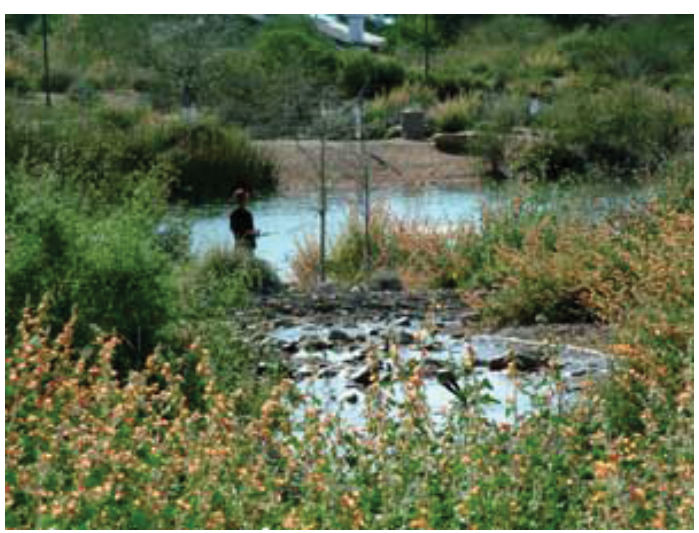

Riparian Preserve at Water Ranch recreational opportunities. This preserve allows the city of Gilbert to reuse $100 \%$ of its wastewater through a series of recharge basins that support marshland, native riparian, and upland vegetation areas. The site includes more than 4.5 miles of trails. Our visit will include a tour led by naturalist Jennie Rambo and water recharge operations expert Patty Jordan. Participants will have the opportunity to observe the riparian habitat's ecological benefits for birds and native vegetation and its utility as a natural reservoir.

\section{EVENTS}

Visit https://annual.ametsoc.org/index.cfm/2019/programs/events/ for a complete list of all of the events taking place in Phoenix. 


\section{Accommodations}

annual.ametsoc.org/index.cfm/2019/travel/hotels/

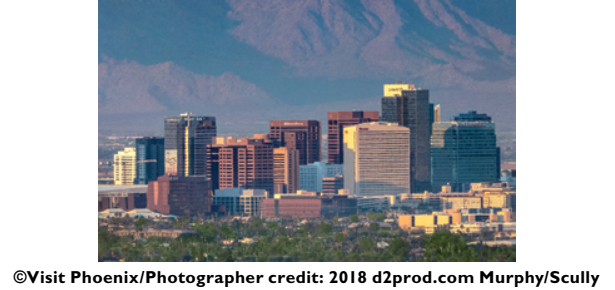

\section{Transportation}

www.visitphoenix.com/learn-plan/getting-around/

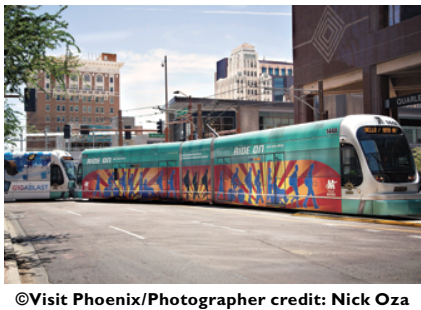

\section{Food and Entertainment}

www.visitphoenix.com/american-meteorological -society/

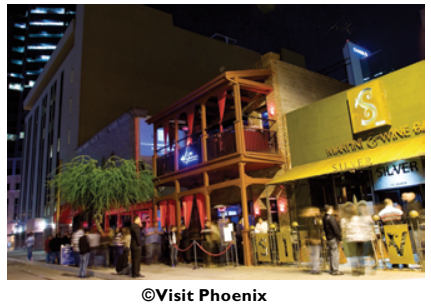

\section{Student Support Opportunities}

Many conferences offer travel awards as well as award certificates and cash prizes for the best student oral and poster presentations at the conference. Check out this page for specifics: https://annual.ametsoc.org/2019/index.cfm /information-for/students/student-award -opportunities/

\section{Baby Gear Rental}

For those attendees traveling to the meeting with a baby or toddler, local baby rental gear company 3 Peas Baby Gear is offering a $10 \%$ discount on rentals. Use code "AMS" when booking.

\section{Quiet Room}

Looking for a quiet place to relax in between sessions? For the first time, AMS will have open a quiet room available at the Annual Meeting. Room 206 in the West Building and will be available Monday-Thursday for those seeking a moment of quiet of reflection.

\section{INCLUSIVITY AT THE 99TH ANNUAL MEETING}

The American Meteorological Society (AMS) is committed to, and benefits from, the full and equitable participation of a diverse community in its membership, in its activities, and in the audiences that it serves.

This year the AMS is celebrating the Annual Meeting theme "Understanding and Building Resilience to Extreme Events by Being Interdisciplinary, International, and Inclusive (III)" with several new activities. Meeting attendees will find that welcome signs are in the 12 most commonly spoken languages of attendees to past AMS meetings; volunteers who speak more than one language will be serving at information booths and as AMS Beacons. Weatherfest will have bilingual volunteers (English-Spanish) and sign-language interpreters available to make the event welcoming to as many visitors as possible. Any meeting participant will be able to honor the languages they speak by picking up a bilingual ribbon at the registration desk with a sticker of the language of their choice. Exhibitor staff with multiple language skills are encouraged to participate. For attendees interested in bilingual science communication and outreach, there will be a session on "Bilingual Science Outreach and Communication: Efforts by the Broadcast Meteorology Community, Informal Education Community, and Various Organizations to Broaden the Field in the Earth and Atmospheric Sciences," as part of the Seventh Symposium on Building a Weather-Ready Nation: Enhancing Our Nation's Readiness, Responsiveness, and Resilience to High-Impact Weather Events. 


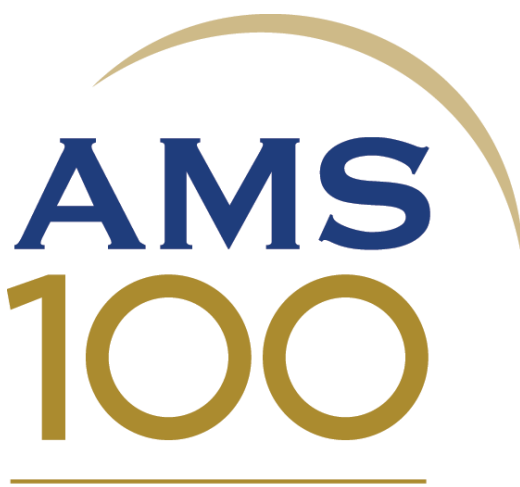

ADVANCING SCIENCE SERVING SOCIETY

\section{Centennial at the Annual}

The AMS is kicking off its Centennial celebration at the 99th Annual Meeting. Here is a list of the ways meeting attendees can take part in Phoenix!

- Watch a Centennial presentation

- Visit the AMS Resource Center

- Preview the AMS Timeline

- Take a Centennial selfie

- Share your story in a five-to-ten-minute interview

Want to know more about the AMS Centennial Celebration? Check out the webpage here: https://www.ametsoc.org/index.cfm/amsI00/ and on twitter \#AMS100.

\section{CENTENNIAL PRESENTATIONS}

\begin{tabular}{|c|c|c|c|}
\hline Conference & Session Title & Paper Title & $\begin{array}{l}\text { Session Day, } \\
\text { Paper Start-End Time }\end{array}$ \\
\hline $\begin{array}{l}\text { I5th Annual Symposium on } \\
\text { New Generation Operational } \\
\text { Environmental Satellite Systems }\end{array}$ & $\begin{array}{l}\text { The Evolution of Joint Polar } \\
\text { Satellite System: How Polar } \\
\text { Satellites Transformed Weather } \\
\text { Forecasting }\end{array}$ & $\begin{array}{l}\text { The Evolution of Joint Polar } \\
\text { Satellite System: How Polar } \\
\text { Satellites Transformed Weather } \\
\text { Forecasting }\end{array}$ & $\begin{array}{l}\text { Sunday, } 7 \text { January } 2019 \text {, } \\
\text { 8:30-9:00 A.M. }\end{array}$ \\
\hline $\begin{array}{l}\text { Special Symposium on } \\
\text { Mesoscale Meteorological } \\
\text { Extremes: Understanding, } \\
\text { Prediction, and Projection }\end{array}$ & $\begin{array}{l}\text { Mesoscale Meteorology: } \\
\text { Connecting Weather and } \\
\text { Climate Research }\end{array}$ & $\begin{array}{l}\text { Mesoscale Meteorology: } \\
\text { Connecting Weather and } \\
\text { Climate Research }\end{array}$ & $\begin{array}{l}\text { Sunday, } 7 \text { January } 2019 \text {, } \\
\text { 8:45-9:15 A.M. }\end{array}$ \\
\hline 17th History Symposium & $\begin{array}{l}\text { The AMS Historical Research } \\
\text { Network: An Opportunity for } \\
\text { the AMS Centennial and the } \\
\text { Society's Second Century }\end{array}$ & $\begin{array}{l}\text { The AMS Historical Research } \\
\text { Network: An Opportunity for } \\
\text { the AMS Centennial and the } \\
\text { Society's Second Century }\end{array}$ & $\begin{array}{l}\text { Sunday, } 7 \text { January } 2019 \text {, } \\
\text { 10:30-10:45 A.M. }\end{array}$ \\
\hline $\begin{array}{l}\text { Ninth Symposium on Lidar } \\
\text { Atmospheric Applications }\end{array}$ & $\begin{array}{l}\text { Lidar: A 50-Year Personal } \\
\text { Retrospective }\end{array}$ & $\begin{array}{l}\text { Lidar: A 50-Year Personal } \\
\text { Retrospective }\end{array}$ & $\begin{array}{l}\text { Sunday, } 7 \text { January } 2019 \text {, } \\
\text { 2:00-2:30 P.M. }\end{array}$ \\
\hline $\begin{array}{l}\text { Ninth Symposium on Lidar } \\
\text { Atmospheric Applications }\end{array}$ & $\begin{array}{l}\text { From LITE to CALIPSO and } \\
\text { Beyond: A Brief History of } \\
\text { NASA Langley Research Center } \\
\text { Spaceborne Lidar Missions and } \\
\text { Measurements }\end{array}$ & $\begin{array}{l}\text { From LITE to CALIPSO and } \\
\text { Beyond: A Brief History of } \\
\text { NASA Langley Research Center } \\
\text { Spaceborne Lidar Missions and } \\
\text { Measurements }\end{array}$ & $\begin{array}{l}\text { Sunday, } 7 \text { January } 2019 \text {, } \\
\text { 3:00-3:30 P.M. }\end{array}$ \\
\hline 28th Symposium on Education & $\begin{array}{l}\text { Maury Project-25 Years } \\
\text { Supporting Teacher Professional } \\
\text { Development in Physical } \\
\text { Oceanography }\end{array}$ & $\begin{array}{l}\text { Maury Project-25 Years } \\
\text { Supporting Teacher Professional } \\
\text { Development in Physical } \\
\text { Oceanography }\end{array}$ & $\begin{array}{l}\text { Sunday, } 7 \text { January } 2019 \text {, } \\
\text { 3:30-4:00 P.M. }\end{array}$ \\
\hline $\begin{array}{l}\text { Ninth Symposium on Lidar } \\
\text { Atmospheric Applications }\end{array}$ & $\begin{array}{l}\text { Five Decades of Doppler Wind } \\
\text { Lidar Measurements from } \\
\text { Turbulence to Global Scales } \\
\text { (invited presentation) }\end{array}$ & $\begin{array}{l}\text { Five Decades of Doppler Wind } \\
\text { Lidar Measurements from } \\
\text { Turbulence to Global Scales } \\
\text { (invited presentation) }\end{array}$ & $\begin{array}{l}\text { Sunday, } 7 \text { January } 2019 \\
\text { 3:30-4:00 P.M. }\end{array}$ \\
\hline $\begin{array}{l}\text { 35th Conference on } \\
\text { Environmental Information } \\
\text { Processing Technologies }\end{array}$ & $\begin{array}{l}\text { The History of IIPS/EIPT: } \\
\text { Where Did It All Begin? }\end{array}$ & $\begin{array}{l}\text { The History of IIPS/EIPT: } \\
\text { Where Did It All Begin? }\end{array}$ & $\begin{array}{l}\text { Sunday, } 7 \text { January } 2019 \text {, } \\
\text { 4:00-6:00 P.M. }\end{array}$ \\
\hline $\begin{array}{l}\text { I5th Annual Symposium on } \\
\text { New Generation Operational } \\
\text { Environmental Satellite Systems }\end{array}$ & $\begin{array}{l}\text { Monitoring Tropical Cyclones } \\
\text { from Satellite: Improvements } \\
\text { from } 1966 \text { to } 2018\end{array}$ & $\begin{array}{l}\text { Monitoring Tropical Cyclones } \\
\text { from Satellite: Improvements } \\
\text { from } 1966 \text { to } 2018\end{array}$ & $\begin{array}{l}\text { Tuesday, } 9 \text { January } 2019 \text {, } \\
\text { 8:30-9:00 A.M. }\end{array}$ \\
\hline
\end{tabular}

\section{Want to Know More?}

Visit the 99th Annual Meeting website: https://annual.ametsoc.org/index.cfm/2019/. 


\section{CENTENNIAL SESSIONS}

\begin{tabular}{|lll|}
\hline & & Session Day, \\
Conference Title & Session Title & Paper Start-End Time \\
\hline $\begin{array}{l}\text { I4th Symposium on Societal Applications: } \\
\text { Policy, Research, and Practice }\end{array}$ & $\begin{array}{l}\text { The Other Uncertainty: Social, Political, and Cultural } \\
\text { Forms of Uncertainty in Weather Contexts-Part I }\end{array}$ & 7 January, 2019 8:30-10:00 A.M. \\
\hline $\begin{array}{l}\text { 14th Symposium on Societal Applications: } \\
\text { Policy, Research, and Practice }\end{array}$ & $\begin{array}{l}\text { The Other Uncertainty: Social, Political, and Cultural } \\
\text { Forms of Uncertainty in Weather Contexts-Part II }\end{array}$ & 7 January, 2019 10:30-12:00 P.M. \\
\hline $\begin{array}{l}\text { I4th Symposium on Societal Applications: } \\
\text { Policy, Research, and Practice }\end{array}$ & $\begin{array}{l}\text { The Other Uncertainty: Social, Political, and Cultural } \\
\text { Forms of Uncertainty in Weather Contexts-Part III }\end{array}$ & January 2019 2:00-4:00 P.M. \\
\hline $\begin{array}{l}\text { Ninth Symposium on Lidar Atmospheric } \\
\text { Applications }\end{array}$ & Historical Perspectives on Lidar & 7 January, 2019 2:00-4:00 P.M. \\
\hline
\end{tabular}

\section{REGISTRATION}

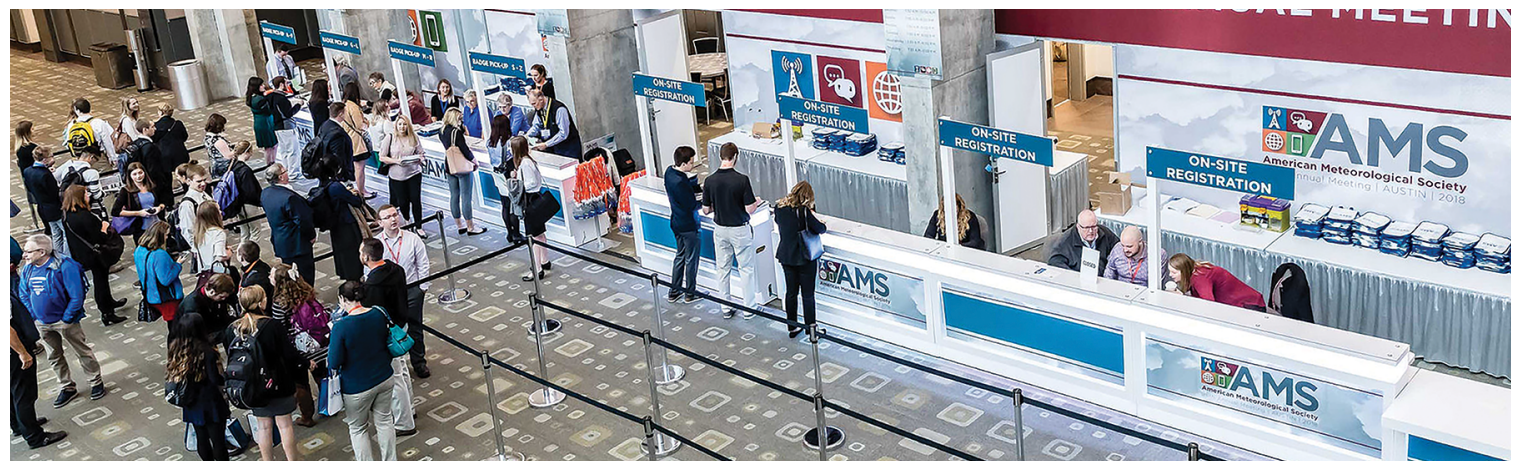

Registration for the AMS 99th Annual Meeting is open online! View rates and special conference registration information below, and register today!

\section{Online Annual Meeting Registration:}

https://www.ametsoc.org/meet/atreg/

\section{Preregistration and Registration Policies}

Everyone presenting (both oral and poster) and/or attending the 99th AMS Annual Meeting must register and wear a badge. Early registration rates are valid through Monday, 3 December 2018. Refunds (less a $\$ 25$ processing fee) will be granted only for cancellations received before 21 December 2018.

Attendees who are unable to complete their registration online should contact the AMS Meetings Department (meetings@ametsoc.org).

By registering for the AMS Annual Meeting, attendees are agreeing to adhere to the Professional and Respectful Conduct at AMS Meetings policy.

Abstract Fee: Please note that the abstract fee of $\$ 95$ is refundable only if the abstract is not accepted.

\section{Guest Program}

AMS is rolling out a new and improved guest program, which will be open to all guests who are coming to Phoenix with 99th Annual Meeting attendees. Updates will be avilable online: https:// annual.ametsoc.org/index.cfm/2019/information-for Iguests.

In the meantime, attendees are encouraged to add their guest's name and e-mail address during the registration process so that they both receive notifications regarding the exciting opportunities AMS has planned.

\section{Additional Banquet Tickets}

An additional Annual Meeting Banquet ticket may be purchased for $\$ 50$ during registration. Child tickets for the Annual Meeting Banquet may be purchased by contacting Christine Card (ccard@ametsoc.org).

\section{REGISTRATION DESK}

The AMS Registration Desk is located on the lower level of the Phoenix Convention Center and will be open for the AMS Student Conference and this year's two-day short courses on Saturday, 5 January, 7:30 A.M.-8:00 P.M.

Annual Meeting registration will be held on

Sunday, 6 January, 7:00 A.M. $-8: 30$ P.M. Monday-Wednesday, 7-9 January, 7:30 A.M.-6:00 P.M. Thursday, 10 January, 7:30 A.M.-3:00 P.M. 


\section{Short Course Registration}

All short course/workshop attendees must register and wear a badge/ribbon. Short course/workshop registration is not included in the 99th Annual Meeting registration, and short course/workshop registration does not include registration for the 99th AMS Annual Meeting.

\section{Press Registration}

The AMS operates a Press Room each year at the Annual Meeting, which provides an excellent opportunity for media with a plethora of presentations, special programs, and exhibits. Eligibility for press registration is limited to the working press and freelance science writers with appropriate identification, as well as public information officers of scientific societies, educational institutions, and government agencies. Press registration can be completed here: https://annual.ametsoc .org/index.cfm/2019/registration/press-registration/.

Members of the press who register for the meeting agree to the following terms:

- No videotaping within session rooms.

- Press members are expected to adhere to the same guidelines as attendees when it comes to the Professional and Respectful Conduct at AMS Meetings policy.

- Press credentials are provided as a courtesy by the AMS and may be revoked by the AMS if in its sole judgment an attendee's presence at the meeting is in any way disruptive.

\section{Information for Presenters}

All authors should visit the AMS Abstract Author and Presenters page at https://www.ametsoc.org /ams/index.cfm/meetings-events/abstract-author-and -presenter-information/ams-presenter-guidelines/ to learn more about managing their abstracts online and presenting their work at an AMS Meeting. For more detailed information about presenting at the 99th Annual Meeting, please visit https://annual.ametsoc .org/index.cfm/2019/information-for/authors-and -presenters/. Important dates for the Presenter's Corner:

Edit abstract title and author list: 1 November Upload presentation before meeting: 3 December Upload supplementary information: 22 February

Login details for the Presenter's Corner may be found in the presenter's abstract initiation and abstract acceptance notification e-mails. To have these e-mails resent, presenters should contact the Meetings Department.

\section{Presenter's Workshop}

New presenters and those looking to hone your presenting skills are invited to attend the Presenter's Workshop on Monday, 7 January, from 1:30 to 2:30 P.M. This miniworkshop is designed to enable participants to use simple guidelines to create more memorable and effective presentations for conference audiences. Participants will receive instruction on the following: 1) the basic rules for clearly organizating information, 2) how to think about the audience, 3) how to use visual aids well, 4) how to develop a bottom line message, and 5) how to become more confident when presenting. This workshop will be broken into three intense units: learning the basics, developing a message and presentation, and delivering that presentation. This hands-on, immediate application of principles will enable participants to revise their own individual presentations for more effective and rewarding results. Workshop leader: Dr. Susan Jasko (Jasko@calu.edu).

\section{EXHIBITS}

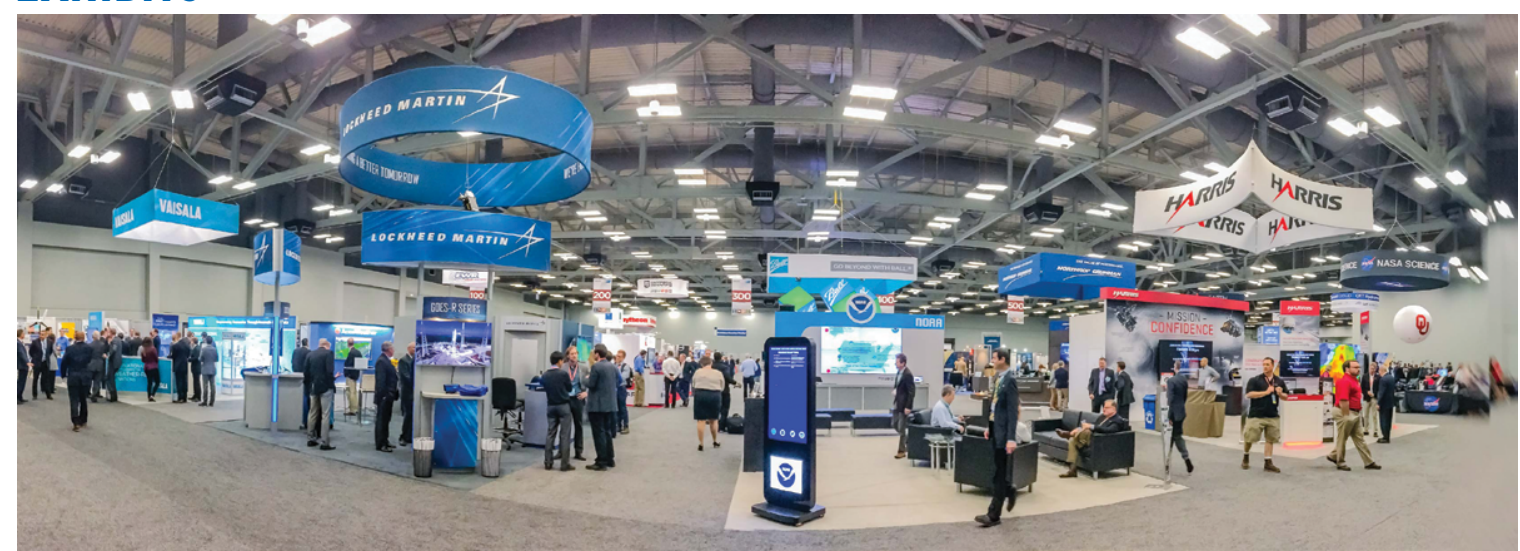

The AMS Annual Meeting is host to the largest exhibit program anywhere in the atmospheric, oceanic, and related sciences. Exhibitors come from all over the United States and abroad showcasing a wide range of products, publications, and services.

Information about the Exhibit Hall: https://annual.ametsoc.org/20/9/index.cfm/exhibits/ Learn how to exhibit at AMS meetings: https:/annual.ametsoc.org/20/9/index.cfm/information-for/exhibitors/ 


\section{AMS $\mid$ Community}

\section{AMS Community Live Demos}

Tuesday, 8 January:

10:00-11:00 A.M.

12:00-1:00 P.M.

4:00-5:00 P.M.

Wednesday, 9 January: $\quad$ 10:00-11:30 A.M.

4:00-5:00 P.M.

Thursday, 10 January: 10:00-11: 00 A.M.

Live demonstrations will be held in the AMS Resouce Center in the Exhibit Hall during the above hours, but all meeting attendees are invited to stop by any time. The Exhibit Hall is open to those looking to discuss/troubleshoot the Community. Attendees interested in scheduling a time to chat about the Community are encouraged to reach out to Jordan Stillman (jstillman@ametsoc.org).

\section{AMS Event Community}

For the 99th AMS Annual Meeting, the AMS Community platform will be hosting a special Event Community, specifically for meeting attendees. The Event Community will be a designated online space where attendees can gather to talk about the meeting - and whatever else comes up on the forum's discussion boards-and connect with fellow attendees ahead of the meeting.

AMS will also be hosting sneak peeks with special guests, sharing meeting information and documents, and sparking meeting-related conversations to help attendees plan and prepare for 99th AMS Annual Meeting in Phoenix. Attendees seeking to keep your finger on the pulse of all things Annual are encouraged to participate.

Attendees will be given the option to join the Event Community when they register for the Annual
Meeting and can remove themselves from the Event Community at any time.

Please contact the Member Community Coordinator at amscommunity@ametsoc.org with any questions or concerns, or to enroll after you have already registered.

https://annual.ametsoc.org/index.cfm/2019 /information-for/all-attendees/annual-meeting -event-community/

\section{AMS BEACONS HELP BRIGHTEN THE AMS ANNUAL MEETING}

$T$ he AMS Beacons Program is rooted in former Executive Director Kenneth Spengler's legacy of fostering the AMS as an open, inclusive, and welcoming organization.

At the 99th AMS Annual Meeting in Phoenix, Beacons will once again be available to assist anyone in attendance-from the first-timer who needs directions to a seasoned attendee who needs some timely and thoughtful advice. Beacons, who may be identified by their bright yellow lanyards, are a volunteer, complementary resource to the AMS staff and are trained on what questions and information should be referred to AMS staff members. For information on how to volunteer as a Beacon, contact Beth Farley, AMS director of Member Services (tel: 617-226-3910; e-mail: bfarley@ametsoc.org).

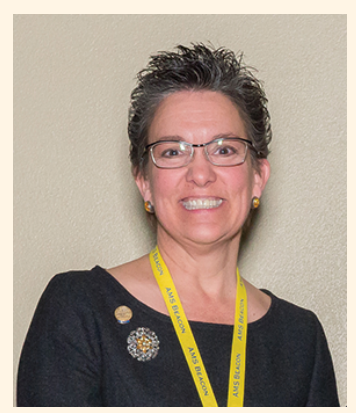

\section{AMS MEETS, TWEETS, AND BLOGS}

W ith more than 3400 presentations to be given at the 2019 AMS Annual Meeting in Phoenix, the best way

to stay up to date with all that is going on is by following all of the AMS social media channels.

Every day during the Annual Meeting, AMS publishes news, interviews, commentaries, updates, photos, and videos across multiple online channels.

The Front Page blog (www.blog.ametsoc.org) extends the reach of attendees and exhibitors beyond the Conference Center walls to fellow members back home and to the general public. In addition to news and commentary during the week in Seattle, the blog features special sessions, news about presenters, tips for attendees, and explores the links between AMS science, the meeting agenda, and the world in the months leading up to the meeting.

In addition to The Front Page, follow the breaking stories and ongoing conversations at the Annual Meeting in real time on Facebook and Twitter. To stay up to date, be sure to "like" AMS on Facebook (www.facebook. com/ametsoc) and follow @a metsoc on Twitter. The Twitter hashtag for the Annual Meeting is \#AMS2019. 


\begin{tabular}{|c|c|c|c|c|c|c|c|c|}
\hline & \multicolumn{4}{|c|}{ BY 3 DECEMBER } & \multicolumn{4}{|c|}{ AFTER 3 DECEMBER } \\
\hline Course Name & Member & $\begin{array}{l}\text { Non- } \\
\text { Member }\end{array}$ & $\begin{array}{l}\text { Student } \\
\text { Member }\end{array}$ & $\begin{array}{l}\text { Student } \\
\text { Non- } \\
\text { Member }\end{array}$ & Member & $\begin{array}{l}\text { Non- } \\
\text { Member }\end{array}$ & $\begin{array}{l}\text { Student } \\
\text { Member }\end{array}$ & $\begin{array}{l}\text { Student } \\
\text { Non- } \\
\text { Member }\end{array}$ \\
\hline $\begin{array}{l}\text { AMS Short Course on Python } \\
\text { for Synoptic Meteorology Using } \\
\text { Metpy [I-day (Sunday) course] }\end{array}$ & $\$ 205$ & $\$ 245$ & $\$ 135$ & $\$ 165$ & $\$ 245$ & $\$ 285$ & $\$ 175$ & $\$ 205$ \\
\hline $\begin{array}{l}\text { An Introduction to Using the } \\
\text { NASA Giovanni System for } \\
\text { Multidisciplinary Research and } \\
\text { Applications [I-day (Sat) course] }\end{array}$ & $\$ 205$ & $\$ 245$ & $\$ 135$ & $\$ 165$ & $\$ 245$ & $\$ 285$ & $\$ 175$ & $\$ 205$ \\
\hline $\begin{array}{l}\text { Applying JPSS Data Products } \\
\text { to Better Forecast Challenging } \\
\text { Weather Events [I-day (Sunday) } \\
\text { course] }\end{array}$ & $\$ 75$ & $\$ 75$ & $\$ 75$ & $\$ 75$ & $\$ 100$ & $\$ 100$ & $\$ 100$ & $\$ 100$ \\
\hline $\begin{array}{l}\text { A Beginner's Course to } \\
\text { Using Python in Climate and } \\
\text { Meteorology [2-day (Saturday- } \\
\text { Sunday) course] }\end{array}$ & $\$ 410$ & $\$ 450$ & $\$ 340$ & $\$ 370$ & $\$ 450$ & $\$ 490$ & $\$ 380$ & $\$ 410$ \\
\hline $\begin{array}{l}\text { Diversity, Equity, and Inclusion } \\
\text { for Geoscientists [I-day (Sunday) } \\
\text { course] }\end{array}$ & $\$ 25$ & $\$ 25$ & $\$ 25$ & $\$ 25$ & $\$ 50$ & $\$ 50$ & $\$ 50$ & $\$ 50$ \\
\hline $\begin{array}{l}\text { GOES-R Series: Forecasting } \\
\text { Applications [I-day (Sunday) } \\
\text { course] }\end{array}$ & $\$ 50$ & $\$ 50$ & $\$ 50$ & $\$ 50$ & $\$ 75$ & $\$ 75$ & $\$ 75$ & $\$ 75$ \\
\hline $\begin{array}{l}\text { Integrating NWP System } \\
\text { Components Using Container } \\
\text { Technology [I-day (Sunday) } \\
\text { course] }\end{array}$ & $\$ 185$ & $\$ 225$ & $\$ 115$ & $\$ 145$ & $\$ 225$ & $\$ 265$ & $\$ 155$ & $\$ 185$ \\
\hline $\begin{array}{l}\text { Introducing the Community } \\
\text { Earth System Model (CESM2) } \\
\text { via an Interactive Hands-On } \\
\text { Tutorial Using the Cloud [1/2-day } \\
\text { (Sunday) course] }\end{array}$ & $\$ 190$ & $\$ 230$ & $\$ 120$ & $\$ 150$ & $\$ 230$ & $\$ 270$ & $\$ 160$ & $\$ 190$ \\
\hline $\begin{array}{l}\text { Machine Learning in Python for } \\
\text { Environmental Science Problems } \\
\text { [I-day (Sunday) course] }\end{array}$ & $\$ 235$ & $\$ 275$ & $\$ 165$ & $\$ 195$ & $\$ 275$ & $\$ 315$ & $\$ 205$ & $\$ 235$ \\
\hline $\begin{array}{l}\text { Tutorial on How to Build a } \\
\text { 3D-Printed Automatic Weather } \\
\text { Station [I-day (Sunday) course] }\end{array}$ & $\$ 240$ & $\$ 280$ & $\$ 170$ & $\$ 200$ & $\$ 280$ & $\$ 320$ & $\$ 310$ & $\$ 240$ \\
\hline $\begin{array}{l}\text { Using NASA High Performance } \\
\text { Computers to Perform Analytics } \\
\text { on Large Earth Science Data } \\
\text { [1/2-day (Sunday) course] }\end{array}$ & $\$ 175$ & $\$ 215$ & $\$ 105$ & $\$ 135$ & $\$ 215$ & $\$ 255$ & $\$ 145$ & $\$ 175$ \\
\hline $\begin{array}{l}\text { Weather and Phased Array } \\
\text { Radar Polarimetry [1/2-day } \\
\text { (Sunday) course] }\end{array}$ & $\$ 160$ & $\$ 200$ & $\$ 90$ & $\$ 120$ & $\$ 200$ & $\$ 240$ & $\$ 130$ & $\$ 160$ \\
\hline
\end{tabular}




\begin{tabular}{|c|c|c|c|c|c|c|}
\hline & \multicolumn{3}{|c|}{$\begin{array}{l}\text { FULL-WEEK PACKAGE } \\
\text { The full-week package includes admission to } \\
\text { all conferences, exhibits, coffee breaks, and } \\
\text { general receptions, plus one Annual Meeting } \\
\text { Banquet ticket. }\end{array}$} & \multicolumn{3}{|c|}{$\begin{array}{l}\text { ONE-DAY PACKAGE } \\
\text { The one-day package includes admission for } \\
\text { one calendar day to all conferences, exhibits, } \\
\text { coffee breaks, and general receptions, with the } \\
\text { exception of the Annual Meeting Banquet. }\end{array}$} \\
\hline Attendee Type & $\begin{array}{l}\text { Early } \\
\text { Registration } \\
\text { Through } 3 \\
\text { Dec }\end{array}$ & $\begin{array}{l}\text { Registration } \\
4 \text { Dec-5 Jan }\end{array}$ & $\begin{array}{l}\text { Late } \\
\text { Registration } \\
\text { On site }\end{array}$ & $\begin{array}{l}\text { Early } \\
\text { Registration } \\
\text { Through } 3 \\
\text { Dec }\end{array}$ & $\begin{array}{l}\text { Registration } \\
4 \text { Dec-5 Jan }\end{array}$ & $\begin{array}{l}\text { Late } \\
\text { Registration } \\
\text { On site }\end{array}$ \\
\hline $\begin{array}{l}\text { AMS Full Member, Affiliate } \\
\text { Member of AMS, Program or } \\
\text { Session Chair }\end{array}$ & $\$ 555$ & $\$ 595$ & $\$ 615$ & $\$ 295$ & $\$ 335$ & $\$ 355$ \\
\hline $\begin{array}{l}\text { Non-Member Speaker or } \\
\text { Poster Presenter, AMS Associate } \\
\text { Member, Exhibitor Attendee, or } \\
\text { Cosponsoring Society Member* }\end{array}$ & $\$ 605$ & $\$ 645$ & $\$ 665$ & $\$ 295$ & $\$ 335$ & $\$ 355$ \\
\hline Non-Member & $\$ 655$ & $\$ 695$ & $\$ 715$ & $\$ 295$ & $\$ 335$ & $\$ 355$ \\
\hline Retired Member & $\$ 195$ & $\$ 235$ & $\$ 255$ & $\$ 90$ & $\$ 130$ & $\$ 150$ \\
\hline AMS Student Member & $\$ 165$ & $\$ 205$ & $\$ 225$ & $\$ 60$ & $\$ 100$ & $\$ 120$ \\
\hline Student Non-Member & $\$ 195$ & $\$ 235$ & $\$ 255$ & $\$ 90$ & $\$ 130$ & $\$ 150$ \\
\hline
\end{tabular}

* The following societies are cosponsoring the 99th AMS Annual Meeting: Air \& Waste Management Association, American Academy of Environmental Engineers and Scientists (AAEES), American Geophysical Union (AGU), American Geosciences Institute (AGI), Australian Meteorological and Oceanographic Society (AMOS), Canadian Meteorological and Oceanographic Society (CMOS), Chinese Meteorological Society (CMS), European Meteorological Society (EMS), Indian Meteorological Society (IMS), International Association of Broadcast Meteorology, National Weather Association (NWA).

\section{SPECIAL CONFERENCE REGISTRATION}

\begin{tabular}{|c|c|c|c|}
\hline Special Conference Name & Early Rate & Late Rate & Link for More Information \\
\hline $\begin{array}{l}\text { I8th Annual AMS Student } \\
\text { Conference and Career Fair }\end{array}$ & $\begin{array}{l}\$ 50 \text { before } \\
15 \text { December }\end{array}$ & $\begin{array}{l}\$ 75 \text { after } \\
15 \text { December }\end{array}$ & $\begin{array}{l}\text { https://annual.ametsoc.org/index.cfm/2019/registration } \\
\text { /special-conference-registration//8th-annual-ams-student } \\
\text {-conference-and-career-fair-registration/ }\end{array}$ \\
\hline $\begin{array}{l}\text { Seventh AMS Conference for } \\
\text { Early Career Professionals }\end{array}$ & $\begin{array}{l}\$ 35 \text { before } \\
15 \text { December }\end{array}$ & $\begin{array}{l}\$ 50 \text { after } \\
15 \text { December }\end{array}$ & $\begin{array}{l}\text { https://annual.ametsoc.org/index.cfm/2019/registration } \\
\text { /special-conference-registration/seventh-ams-conference } \\
\text {-for-early-career-professionals-registration/ }\end{array}$ \\
\hline $\begin{array}{l}\text { 22nd Conference of } \\
\text { Atmospheric Science } \\
\text { Librarians International }\end{array}$ & $\begin{array}{l}\$ 50 \text { before } \\
3 \text { December }\end{array}$ & $\begin{array}{l}\$ 75 \text { after } \\
3 \text { December }\end{array}$ & $\begin{array}{l}\text { https://annual.ametsoc.org/index.cfm/20I9/registration } \\
\text { /special-conference-registration/22nd-conference-of } \\
\text {-atmospheric-science-librarians-international-registration/ }\end{array}$ \\
\hline
\end{tabular}


Booth 429, Exhibit Hall 5, will host the AMS Resource Center, which provides information about AMS membership and programs, including journals and books; certifications; precollege and college education initiatives; student opportunities; the AMS Policy Program; local chapters; AMS meetings; and the AMS Community. AMS provides many opportunities for everyone across the entire field, whether just starting out or with years of experience. Meeting attendees are invited to stop by and learn more about AMS and the Society's community of researchers, scientists, broadcasters, authors, educators, and other professionals, students, and weather enthusiasts. Help celebrate the AMS Centennial kickoff on Monday evening (there will be cake!).

\section{Book Talks @ Authors Corner}

Come to the "AMS Books Authors Corner" at the Resource Center in the Exhibit Hall during poster breaks (Tuesday and Wednesday 4:006:00 P.M.) for engaging discussions on some of the most influential meteorologists, storms, advances, and impacts of the last 100 years. A schedule of speakers will be announced at the meeting.
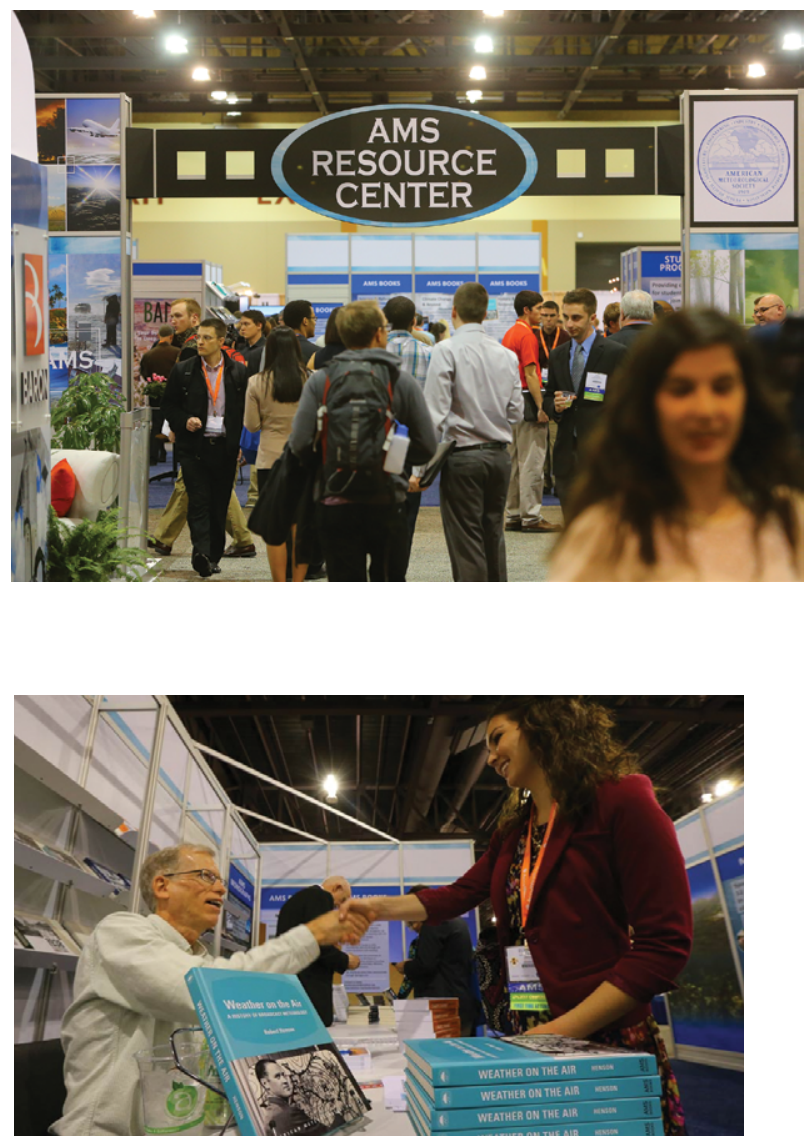

\section{Meet the President}

Tuesday, Wednesday, and Thursday, 8-10 January 2019, 10:00-10:30 A.M., Phoenix Convention Center, Exhibit Hall I

All are encouraged to stop by the AMS Resource Center in the Exhibit Hall to meet AMS President Roger M. Wakimoto.

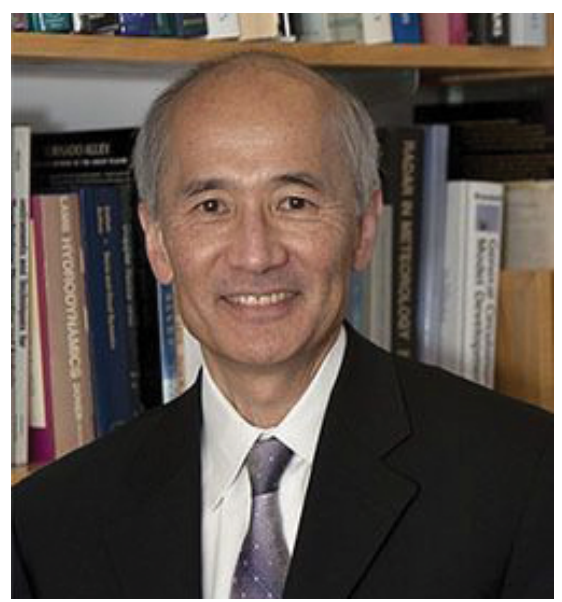

